\title{
Unsteady Reynolds averaged Navier-Stokes simulation of the post-critical flow around a closely spaced group of silos
}

\author{
J. Hillewaere $^{\mathrm{a}, *}$, D. Dooms ${ }^{\mathrm{a}}$, B. Van Quekelberghe ${ }^{\mathrm{b}}$, \\ J. Degroote $^{\mathrm{b}}$, J. Vierendeels ${ }^{\mathrm{b}}$, G. De Roeck ${ }^{\mathrm{a}}$, G. Lombaert ${ }^{\mathrm{a}}$, \\ G. Degrande ${ }^{a}$ \\ ${ }^{a}$ Department of Civil Engineering, K.U.Leuven, \\ Kasteelpark Arenberg 40, B-3001 Leuven, Belgium \\ ${ }^{\mathrm{b}}$ Department of Flow, Heat and Combustion Mechanics, Ghent University, \\ St.Pietersnieuwstraat 41, B-9000 Ghent, Belgium
}

\begin{abstract}
During a storm in October 2002, wind induced ovalling vibrations were observed on several empty silos of a closely spaced group (pitch-to-diameter ratio of 1.05) consisting of 8 by 5 silos in the port of Antwerp (Belgium). Numerical simulations of the turbulent wind flow are performed to clarify the occurrence of the observed ovalling vibrations near the lee side corner of the group by studying the dynamic wind pressures on the silo surfaces and linking to the dynamic properties of the silo structures. As the orientation of the group largely affects the pressure distribution around the cylinders of the group, the influence of the angle of incidence of the wind flow on these ovalling vibrations is examined while other parameters, such as spacing ratio and Reynolds number are unchanged. To achieve results within a rea-
\end{abstract}


sonable computation time, 2D unsteady Reynolds averaged Navier-Stokes (URANS) equations using Menter's shear stress transport turbulence model were performed. In order to elucidate the influence of the applied turbulence model and to qualitatively validate the spatial and temporal discretization of the $2 \mathrm{D}$ highly turbulent post-critical $\left(\operatorname{Re}=1.24 \times 10^{7}\right)$ flow simulations for the silo group, single cylinder simulations were used. The geometric resemblance of the group arrangement with rectangular cylinders on the one hand and of the interstitial spaces with tube arrays (e.g. heat exchangers) on the other hand is used to qualitatively compare the observed flow phenomena. The simulations show that the silo group can be treated neither as a tube array nor as a solid bluff body. Subsequent linking of dynamic wind pressures to dynamic properties of the silo structures reveals strong narrow band frequency peaks in the turbulent pressure coefficient spectra of the silos near the lee side corners of the group that match the structural natural frequencies of the third and fourth ovalling mode shape of the silos. This match indicates a forced, resonant response which corresponds with the observed pattern of ovalling vibrations with three and four circumferential wavelengths. While the precise physical excitation mechanism is not yet fully understood, the simulations exclude discrete vortex shedding and since fluidelastic instability could not be considered, only turbulent buffeting remains which could very well give rise to the narrow band wake phenomena causing the ovalling silo wall vibrations.

Key words: circular cylinder, cylinder array, ovalling, silo group, post-critical flow, wind induced vibration

* Corresponding author. Tel.: +32 163216 77; fax: +32 16321988 .

Email address: jeroen.hillewaere@bwk.kuleuven.be (J. Hillewaere).

URL: http://www.kuleuven. be/bwm (J. Hillewaere). 


\section{Introduction}

Circular cylinders are encountered in numerous civil engineering constructions as silos, chimneys, water towers, power transmission lines, offshore structures and suspension bridge cables. Wind loading is an important design load for these structures since transient wind loads may induce unexpected instabilities, possibly leading to structural failure. During a storm in October 2002, ovalling was observed on several empty silos near the lee side corner of a group consisting of forty silos in the port of Antwerp (Belgium). Wind induced ovalling vibrations are an aeroelastic phenomenon where the cross section of the structure deforms as a shell without bending deformation with respect to the longitudinal axis of symmetry (Païdoussis et al., 1982). Hence, empty silos are more susceptible to ovalling while shell deformations of filled silos are suppressed.

Basic time averaged wind load cases as described in standards, e.g. Eurocode 1 - Part 1.4 (BIN, 2005), are unable to explain the mentioned transient aeroelastic silo vibrations. A more realistic estimation of the forces and pressure distributions on the silo group is required, especially since the specific group arrangement drastically changes the fluid flow around the group. Computational fluid dynamics (CFD) is an interesting alternative to more expensive wind tunnel tests or in situ experiments to get a thorough understanding of the fluid flow around such groups.

In the present paper, numerical simulations of the turbulent wind flow, modelled as incompressible (low Mach number) flow, are performed to clarify the occurrence of the observed ovalling vibrations at the lee side corner silos of 
the group. By studying the dynamic wind pressures on the silo surfaces and linking them to the dynamic properties of the silo structures, it is investigated if ovalling vibrations can be excited in the silo group. No feedback of structural displacements to the fluid flow is included. The influence of the angle of incidence $\alpha$ of the wind flow on these ovalling vibrations is examined. Other parameters such as the spacing ratio and the Reynolds number are unchanged.

The outline of this paper is as follows. Details on the observed ovalling vibrations and the structural properties of the silo group are discussed in the next section. The applied computational model and assumptions are discussed in the third section. The choice of a particular numerical method is very important in CFD simulations and is therefore carefully explained. For validation of the applied numerical procedure, the less complex and better documented case of two-dimensional flow around a single silo in the post-critical regime is considered. The results are compared with recommendations from standards, experimental data and the few numerical results of both two-dimensional (2D) and three-dimensional (3D) simulations available in literature. In the fourth section, the complex flow around the silo group is analysed. The flow is calculated for a range of angles of incidence $\alpha$ of the wind flow between $0^{\circ}$ and $90^{\circ}$. Similarities between the present wind flow, the flow within tube arrays (e.g. heat exchangers) and the flow around rectangular cylinders are discussed. Finally, the fluctuating pressure distributions on the silos are examined and linked to the dynamic properties of the silos in order to elucidate the influence of the angle of incidence on the magnitude and location of the observed ovalling vibrations. 


\section{Observations and structural properties of the silo group}

The forty silos are placed in five rows of eight silos with gaps of $0.3 \mathrm{~m}$ between two neighbouring silos (figures 1 and 2). The silos are circular cylindrical shell structures with a diameter $D=5.5 \mathrm{~m}$ and a height $h=25 \mathrm{~m}$ while the bottom of the silos is located at $16.66 \mathrm{~m}$ above ground level (Dooms et al., 2006). The spacing ratio of the pitch $P$ (i.e. distance between the centres of two cylinders) to the cylinder diameter $D$ is $P / D=1.05$.

[Fig. 1 about here.]

[Fig. 2 about here.]

The ovalling mode shapes and eigenfrequencies of a separate silo have been determined. Each ovalling mode shape is referred to by a couple $(m, n)$ where $m$ denotes the half wave number in the axial direction and $n$ is the number of circumferential waves (Dooms et al., 2006), as clarified in figure 3. Only the mode shapes corresponding to the lowest eigenfrequencies $f_{\text {eig }}$ are shown. Higher eigenmodes would require more energy and are less easily excited.

[Fig. 3 about here.]

During the storm in October 2002, ovalling was observed on several silos near the lee side corner of the silo group (i.e. silo 40 and neighbouring silos 24, $32,38,39$, etc.). The global wind direction at the time of ovalling was at an angle of incidence of approximately $\alpha=30^{\circ}$. The visually detected pattern of oscillations seems to have excited ovalling mode shapes $(1,3)$ and $(1,4)$. During normal wind loading, measurements have furthermore shown that eigenmodes with $n=3$ or $n=4$ have the highest contribution to the response of the silos 
(Dooms et al., 2006).

Because the specific atmospheric conditions during the storm near the silo group were not monitored, approximative wind conditions have to be set up. The location of the silo group, in proximity of the river Scheldt and in vast and flat surroundings, is classified in terrain category II of Eurocode 1 (BIN, 2005). Based on this standard, a mean wind velocity $v_{\infty}=31.8 \mathrm{~m} / \mathrm{s}$ at half the height of the silo, at $30 \mathrm{~m}$ above ground level is found. The highly turbulent regime of the flow around the group of silos is hence post-critical at Reynolds number $\operatorname{Re}=v_{\infty} D / \nu=1.24 \times 10^{7}$ (Zdravkovich, 1997). No experimental or numerical data applicable to this case are available in the literature because of the specific character of the array with such a small spacing ratio and the present high Reynolds number.

\section{Computational model}

The simulation of highly turbulent flows around complex geometries is a challenging task, requiring simplifications in the computational model. In order to verify the accuracy and to validate the applied numerical procedure, the case of a single cylinder in cross flow is also calculated. This approach, known as the Building Block Approach and introduced by the AIAA (Versteeg and Malalasekara, 2007), allows the proposed computational model to be validated for the case of a simpler system for which experimental data are available. The lack of experimental data for the 8 by 5 silo group makes this approach particularly interesting. The same numerical procedure and mesh design is applied for both the single cylinder and the cylinder group simulations. 


\subsection{Numerical procedure}

The finite volume method is used for the discretization of the incompressible Navier-Stokes equations in the CFD simulations. Since it is unnecessary for engineering purposes to resolve the details of turbulent fluctuations in the flow, techniques have been developed for the numerical treatment of turbulence, e.g. the Reynolds averaged Navier-Stokes (RANS) procedure, large eddy simulations (LES), detached eddy simulations (DES) or direct numerical simulation (DNS). The choice of a particular technique depends on the complex geometry, the highly turbulent character of the flow and the computation time. While the time required for RANS computations is modest, it is well known that conventional LES is extremely expensive at high Reynolds numbers since the small but dynamically important near-wall flow structures have to be solved numerically (Catalano et al., 2003). It is also important to assess whether the chosen solution method is capable of solving the specific fluid problem. Moulinec et al. (2004) for example warn that some phenomena present in complex flows around tube bundles cannot be predicted by RANS models while Ong et al. (2009) concluded that RANS gives satisfactory qualitative agreement with published experimental data and numerical results for the flow around a smooth circular cylinder at very high Reynolds numbers. When applying the unsteady Reynolds averaged Navier-Stokes (URANS) approach in the present simulations, physical validation of the results will therefore have to be carefully executed.

In order to compute the flow phenomena leading to ovalling vibrations in the silo group within a reasonable time, a $2 \mathrm{D}$ cross section of the silo group will be considered. Although the flow around the entire silo group and its wake 
is clearly $3 \mathrm{D}$, the flow will be much more $2 \mathrm{D}$ on the smaller scales around the separate closely spaced silos at the lee side of the group. Moreover, 3D flow simulations over complex bodies remain computationally very expensive and are therefore limited to moderate Reynolds numbers while 2D simulations are quite feasible, even for complex geometries and relatively high Reynolds numbers.

In the general RANS procedure, the Navier-Stokes equations (continuity and momentum equations) are modified through the procedure of Reynolds averaging. The velocity field is split into an averaged and a fluctuating part and two new variables are introduced in the equations: the turbulent kinetic energy $k$ and the kinematic turbulent viscosity $\nu_{\mathrm{t}}$ that is often rewritten as the turbulent dissipation $\varepsilon$ or the specific dissipation rate $\omega$. Two additional transport equations, for either $k$ and $\varepsilon$ or $k$ and $\omega$, are added to the set of governing equations. Menter (1994) suggests a hybrid shear-stress transport (SST) turbulence model, blending the robust and accurate formulation of the $k-\omega$ model in the near-wall region with the free stream independence of the $k-\varepsilon$ model in the far field, making it reliable for a wider class of flows. Since the present study is primarily concerned with determining dynamic pressure loads on the silo walls, this SST model will be used because of its efficient near-wall modelling.

To solve the resulting discretized set of equations, a second order interpolation of the pressure, a second order upwind interpolation of the momentum, the turbulent kinetic energy $k$ and the specific dissipation rate $\omega$ are applied, while a second order implicit, unconditionally stable, time stepping method is used. A coupled pressure-based calculation is performed to deal with the pressure-velocity coupling between the momentum and the continuity equa- 
tions. Unlike segregated algorithms such as PISO (Issa, 1986) or SIMPLE (Patankar and Spalding, 1972), the pressure-based coupled algorithm solves a coupled system of equations consisting of the momentum equations and the pressure based continuity equation, improving significantly the rate of convergence. The iterative calculation process for the transient solution is truncated when the normalized residuals for the continuity equation, the momentum equations, the turbulent kinetic energy and the specific dissipation rate reach a level below $10^{-5}$.

The software package Fluent 12.0 (Ansys, 2009) is used for the simulations. The following paragraphs show, through verification and validation, that the use of this commercial software package yields acceptable results for this study.

\subsection{Computational domain and boundary conditions}

The boundaries of the fluid domain should be sufficiently far from the nearwall region where accuracy is important. For the flow around a single cylinder, Behr et al. (1995) suggest a distance of at least $8 D$ to the inlet of the domain and the lateral boundaries and a distance of $22.5 \mathrm{D}$ to the outlet, with $D$ the diameter of the cylinder. Holloway et al. (2004) use similar distances to the boundaries of the domain. As shown in figure 4, distances of $9 D$ and $30 D$ are adopted for the single cylinder case and, equivalently, $9 D_{\mathrm{g}}$ and $30 D_{\mathrm{g}}$ for the group configuration, where $D_{\mathrm{g}}$ represents the projected width of the silo group (figure 2). The group is rotated in the flow to simulate different angles of incidence.

[Fig. 4 about here.] 
For the spatial discretization, the computational domain is divided in three zones, as shown in figure 4 for the single cylinder case. Zone 1 is located close to the rigid body, where a highly refined body-fitted grid arrangement is applied to calculate the near-wall behaviour with sufficient accuracy. In zone 2 , located in the wake of the rigid body, the refinement of the non-orthogonal grid gradually decreases towards the outlet of the domain. In zone 3, the remainder of the domain, the refinement of the triangular unstructured grid gradually decreases from the center of the domain towards the boundaries. The size of the created finite volume cells depends on the required accuracy. Details on mesh refinement are given in paragraph 3.3.

In the computations, the air density is $\rho=1.25 \mathrm{~kg} / \mathrm{m}^{3}$ and its dynamic viscosity is $\mu=1.76 \times 10^{-5} \mathrm{Pas}$. At the inlet boundary, defined as a velocity inlet with imposed free stream velocity $v_{\infty}=31.8 \mathrm{~m} / \mathrm{s}$, the turbulent energy $k$ and specific dissipation rate $\omega$ have to be defined. A turbulence intensity $\mathrm{Tu}=1 \%$ is assumed so that $k=3\left(v_{\infty} \mathrm{Tu}\right)^{2} / 2=0.152 \mathrm{~J} / \mathrm{kg}$ at the inlet. The turbulence length scale $l$ is chosen as a percentage of a characteristic dimension of the problem (Sak et al., 2007), e.g. $l=0.06 D_{g}=1.8 \mathrm{~m}$ for $\alpha=0^{\circ}$, and hence a dissipation rate $\omega=C_{\mu}^{-1 / 4} \sqrt{k} / l=0.395 \mathrm{~s}^{-1}$ is imposed at the inlet with $C_{\mu}=0.09$ a $k-\epsilon$ model constant. Since Menter's SST turbulence model is based on a blend of the original $k-\omega$ model in the inner region of the boundary layer and the standard $k-\epsilon$ model in the outer region of the flow domain, it is reasoned that for the determination of the boundary conditions at the far ends of the domain the application of this $k-\epsilon$ model constant is justified. The applied inlet turbulence intensity is reasonably low when compared to values up to $20 \%$ on the earth surface while much larger (integral) length scales are found in the atmospheric boundary layer as well (ESDU, 1985). However, 
while large turbulence length scales should be resolved in the simulations, they are not in URANS simulations where turbulence models are used. Imposing higher turbulence intensities associated with large length scales would result in excessive turbulence viscosity of the flow and yield unphysical results. In this study, large turbulence length scales in the atmospheric boundary layer are therefore not considered and a length scale based on the considered geometry is used. The use of these lower turbulence levels is justified in paragraph 4.1 where the effect of turbulence at the inlet of the domain is illustrated. The outlet boundary of the domain is modelled as a pressure outlet where the static pressure is set equal to the reference pressure. At the lateral boundaries symmetry is imposed. The cylinder walls are considered smooth and no-slip boundary conditions are applied.

\subsection{Spatial and temporal grid refinement}

For transient simulations, the governing equations must be discretized in both space and time. The verification of convergence and grid independence is performed for the single cylinder case.

Spatial grid independence is studied by changing the mesh size in the three zones of the computational grid. In all three zones, the optimal grid size was chosen after several stages of grid refinement. The near-wall treatment at the solid cylinder walls is examined by changing the aspect ratio (AR) of the first grid cell next to the wall. Grid refinement in this region affects the dimensionless distance to the wall $y^{+}=v_{\tau} y / \nu$ and the dimensionless velocity $v^{+}=v / v_{\tau}$, with $v_{\tau}=\sqrt{\tau_{w} / \rho}, y$ the distance to the wall and $\tau_{w}$ the wall shear stress. Indeed, when the mesh size is small enough and the wall-adjacent cell 
is located in the viscous sublayer $\left(y^{+}<5\right)$, the velocity $v^{+}$varies linearly with $y^{+}$and the sublayer is explicitly computed with this linear (laminar) law. For larger mesh sizes, when the wall-adjacent cell is located in the range $50<y^{+}<500$, the well known logarithmic wall functions are used to model the near-wall behaviour of the fluid flow:

$$
v^{+}=\frac{1}{\kappa} \ln y^{+}+B
$$

with $\kappa=0.41$ the von Kármán constant and $B=5.2$. When the wall-adjacent cell is located in the buffer layer (range $5<y^{+}<50$ ) the logarithmic wall functions do not apply anymore, nor is it accurate to use the linear (laminar) law. As a result, simulations where the first grid cell is located in the buffer layer are known to yield inaccurate results and should therefore be avoided.

In figure 5 several levels of refinement of the single cylinder mesh are compared and the different near-wall approaches can be clearly discerned. In the computationally most expensive case with $y_{\max }^{+}=4$, the entire boundary layer is numerically resolved. In all other simulations, near-wall behaviour is simulated with logarithmic wall functions, significantly reducing the number of cells and computational time. For four mesh resolutions, the use of the log law is justified $\left(y_{\max }^{+}=97,130,196\right.$ and 375) and convergence is observed. Similar results are found as for the more expensive simulation with $y_{\max }^{+}=4$. In the other cases $\left(y_{\max }^{+}=17,25\right.$ and 49$)$, the wall-adjacent cell is located mainly in the buffer layer, where the logarithmic wall functions are less accurate and diverging results can be observed in figure 5 .

[Fig. 5 about here.]

To minimize computational time and yet retain sound simulation results, the 
mesh with $\mathrm{AR}=50$ and $y_{\max }^{+}=97$ will be applied for all simulations, as shown in figure 6. Since we are mainly interested in determining fluctuating pressures on the cylinder walls, it is most important that amplitude and frequency of time dependent properties (e.g. of drag or lift coefficient) are accurately computed. Comparison of the present mesh $\left(y_{\max }^{+}=97\right)$ with the highly refined mesh $\left(y_{\max }^{+}=4\right)$ yields negligible differences: $1.2 \%$ for the amplitude of drag, $5.2 \%$ for the amplitude of lift and $4.2 \%$ for the frequency of both drag and lift.

[Fig. 6 about here.]

Figure 7 shows the maximal lift coefficient for multiple time step reductions for the mesh with $\mathrm{AR}=50$ and $y_{\max }^{+}=97$. It can be seen that convergence is reached for a time step of approximately $0.00125 \mathrm{~s}$. The computational efforts can be drastically reduced when a slightly larger time step $\Delta t=0.005 \mathrm{~s}$ is used. The relevant physical phenomena will prevail in simulations with this time step, as will be illustrated when validating the simulation results.

[Fig. 7 about here.]

\subsection{Validation of simulation results}

The flow around a circular cylinder has been widely studied, mainly focusing on the vortex shedding in the sub-critical regime and the drag crisis in the critical regime. At post-critical Reynolds numbers, the wake and shear layer are fully turbulent, the boundary layers become fully turbulent prior to separation and coherent vortex shedding appears. Very few experiments or computations have been performed for the cross flow around a cylinder in the 
post-critical regime. A short overview of data available for the validation of the single cylinder simulation results is given.

The pressure coefficient $C_{\mathrm{p}}(\theta, t)$ along the circumference of a cylinder at a certain time $t$ is defined as

$$
C_{\mathrm{p}}(\theta, t)=\frac{p(\theta, t)-p_{\infty}}{\rho v_{\infty}^{2} / 2}
$$

with $p_{\infty}$ the free stream pressure and $v_{\infty}$ the free stream velocity of the fluid. The time averaged pressure coefficient $\bar{C}_{\mathrm{p}}(\theta)$ is calculated as:

$$
\bar{C}_{\mathrm{p}}(\theta)=\frac{1}{T_{\mathrm{vs}}} \int_{t=0}^{T_{\mathrm{vs}}} C_{\mathrm{p}}(\theta, t) d t
$$

with $T_{\mathrm{vs}}$ a vortex shedding period. In table 1, typical flow characteristics are compared for the present simulations and for experiments and simulations from the literature: the Reynolds number Re, the Strouhal number $\mathrm{St}=f_{\mathrm{vs}} L / v_{\infty}$ with $f_{\mathrm{vs}}$ the vortex shedding frequency and $L$ the characteristic length, equal to the diameter $D$ of the cylinder, the separation angle $\theta_{\mathbf{s}}$ (figure 8), the minimum time averaged pressure coefficient $\bar{C}_{\mathrm{p}}^{\text {min }}$ (figure 8), the time averaged base pressure coefficient $\bar{C}_{\mathrm{p}}^{\mathrm{b}}$ (figure 8 ) and the time averaged drag coefficient $\bar{C}_{\mathrm{d}}=\int_{0}^{2 \pi} \bar{C}_{\mathrm{p}}(\theta) \cos \theta d \theta$. For the present simulation at $\operatorname{Re}=1.24 \times 10^{7}$, the time averaged pressure coefficient is shown in figure 8 with $\theta_{\mathrm{s}}=116^{\circ}$ and St $=0.32$.

[Fig. 8 about here.]

Experimental data for high Reynolds number flows around circular cylinders are only scarsely available in literature, e.g. James et al. (1980), Schewe (1983), Shih et al. (1993) and Zan (2008). In an elaborate overview of wind tunnel 
experiments for Reynolds numbers from $0.73 \times 10^{7}$ to $3.65 \times 10^{7}$, gathered by Zdravkovich (1997) (see figure 9), separation occurs between $\theta=100^{\circ}$ and $110^{\circ}$ and Strouhal numbers are between $\mathrm{St}=0.27$ and 0.32 (see table 1 ). For Reynolds numbers larger than $0.5 \times 10^{7}$, smooth flow data of Zan (2008) indicate that the Strouhal number remains at 0.2, whereas Schewe (1983) indicates that it rises to about 0.3 as the Reynolds number approaches a value of $10^{7}$, consistent with the tendency of the Strouhal number to rise from 0.2 to 0.3 in the range of Reynolds numbers between $10^{6}$ and $10^{7}$ (Zdravkovich, 1997). Zan (2008) explains that these differences may be due to a different length-diameter ratio of the model: a larger Reynolds number on a low lengthdiameter ratio model would give rise to an increased Strouhal number. Indeed, James et al. (1980) reported $\mathrm{St}=0.22$ for a model with a length-diameter ratio lower than 5 , while Schewe (1983) found $\mathrm{St} \approx 0.27$ for ratios larger than 10 . The situation is even more complex when the freestream turbulent flow is considered: Zan (2008) reports vortex shedding with $\mathrm{St} \approx 0.25$ independent of the Reynolds number in a flow with $13 \%$ turbulence intensity. The considerable scatter in the experimental data can therefore not only be explained by the difference in Reynolds number, but is also due to free stream turbulence and length-diameter ratio of the model. Furthermore, other differences such as the roughness of the cylinder walls and blocking ratio (i.e. the ratio of the model area to the test section area), which have not been discussed here, also influence the results of wind and water tunnel tests.

Several 2D URANS simulations have been reported in the literature for highly turbulent cross flows around circular cylinders, listed in table 1. Celik and Shaffer (1995) used URANS with an empirically fixed transition point to compute the flow for Reynolds numbers up to $3.6 \times 10^{6}$. The predictions are 
strongly influenced by the grid size, especially in the boundary layer. The best results are obtained when the wall-adjacent cell is located in the viscous sublayer. Holloway et al. (2004) applied URANS simulations for flows with increasing Reynolds numbers up to $10^{7}$, showing that these are capable to correctly predict the boundary layer transitions from laminar to fully turbulent. Saghafian et al. (2003) compared flow computations with the standard $k-\varepsilon$ model and a nonlinear eddy-viscosity model where cubic terms are introduced to account for effects of streamline curvature. Younis and Przulj (2006) modified the $k-\varepsilon$ model by adding a source term in the dissipation rate equation to account for the direct energy input from the mean flow into the random turbulence motions at the vortex shedding frequency. Comparison with the RNG $k-\varepsilon$ model showed that the pressure coefficient along the circumference at Reynolds number $3.5 \times 10^{6}$ significantly decreases down to a value between $150^{\circ}$ and $180^{\circ}$.

Travin et al. (2000) applied 3D DES for Reynolds numbers up to $3 \times 10^{6}$. For turbulent separation cases, the results of 2D URANS computations are very close to the results of $3 \mathrm{D}$ DES. Adding a curvature correction term to the turbulence model improves the estimate of the separation angle, the base pressure and the drag coefficient. Catalano et al. (2003) used 3D LES with the dynamic Smagorinsky model to compute the flow for Reynolds numbers up to $2 \times 10^{6}$ and compared it with $3 \mathrm{D}$ URANS results. The solutions of both LES and URANS show relative insensitivity to the Reynolds number and inaccurate predictions at higher Reynolds numbers which are probably due to poor grid resolution.

[Table 1 about here.] 
All experimental and numerical data from literature, listed in table 1, show considerable scatter due to differences in Reynolds number, applied turbulence model, etc. The separation angle $\theta_{\mathrm{s}}$ is slightly overestimated in $2 \mathrm{D}$ numerical simulations in literature as well as in the present simulations and, probably as a result of this, the minimum averaged pressure coefficient $\bar{C}_{\mathrm{p}}^{\text {min }}$ on the cylinder circumference is overestimated as well. However, the main interest in this paper is to estimate fluctuating quantities reasonably well by numerical simulation (e.g. St, pressure fluctuations on cylinder walls). It can therefore be concluded that reasonable agreement is found between the present simulations $(\mathrm{St}=0.32)$ and data from literature (table 1$)$. Figure 9 shows that fluctuations between minimal and maximal pressure coefficients on the circumference of the cylinder $\left(y^{+}=97\right)$ agree reasonably well with the experimental data gathered by Zdravkovich (1997) and Shih et al. (1993). Although it is difficult to compare all parameters, it is concluded that, for the present purposes, satisfactory agreement is found between the present calculations at $\operatorname{Re}=1.24 \times 10^{7}$ and available data.

[Fig. 9 about here.]

\section{Turbulent air flow around the 8 by 5 cylinder group}

The turbulent air flow around the 8 by 5 silo group is simulated for 7 angles of incidence between $0^{\circ}$ and $90^{\circ}$ in steps of $15^{\circ}$. In order to clarify the ovalling vibrations at the lee side corner silos of the group, a distinction is made between vibrations related to the periodicity of the flow within the array and vibrations caused by the large vortex structures behind the entire cylinder bundle. Therefore, both the flow pattern around the cylinder group and the flow in the 
interstitial spaces between the cylinders are discussed separately. The former is based upon the literature on fluid flows around rectangular cylinders and the latter on fluid flow through tube bundles (e.g. in heat exchangers).

\subsection{Computational domain and boundary conditions}

The computational domain and grid are built up similarly to the single cylinder numerical model. Since the separation structures in the wake of the group configuration will be larger, it is expected that the time step $(\Delta t=0.005 s)$ and mesh size $\left(\mathrm{AR}=50\right.$ and $y_{\max }^{+}=102$ for the silo group) as used for the single cylinder will yield sufficiently accurate results for the entire group. Simulations have been performed for an incidence angle $\alpha=30^{\circ}$ to verify this assumption. As expected, it was found that a smaller time step $\Delta t=0.00125 \mathrm{~s}$ does not significantly change the results. Changing the aspect ratio in the near-wall region inevitably changes the modelling of the boundary layer (log-law wall functions vs. modelling of viscous sublayer). It was found that modelling wall functions for $y_{\max }^{+}=102$ yields good results when compared to simulations with very low $y^{+}$where the viscous sublayer is resolved. The wall function approach is not only much less time consuming, but also more reliable because a mesh sufficiently refined in the near-wall region to resolve the entire viscous sublayer is not easily generated for the entire group. The mesh for an angle of incidence $\alpha=30^{\circ}$ with $y_{\max }^{+}=102$ is shown in figure 10 .

[Fig. 10 about here.]

The same boundary conditions as for the single cylinder simulations have been applied for the group simulations. As mentioned in paragraph 3.2, the effect of 
turbulence intensity at the velocity inlet of the domain has been investigated for an incidence angle $\alpha=30^{\circ}$. When turbulence intensities associated with large turbulence length scales as high as $\mathrm{Tu}=10 \%$ are imposed, turbulence viscosity increases. The incident flow acts as a much more viscous fluid in the numerical simulations, altering for instance corner silo drag and lift, as shown in figures 11 and 12. As expected, changing the inlet turbulence intensity from $\mathrm{Tu}=1 \%$ to $\mathrm{Tu}=0.1 \%$ does not affect the results significantly.

[Fig. 11 about here.]

[Fig. 12 about here.]

\subsection{Flow around the cylinder group}

The flow pattern around the 8 by 5 silo group was calculated for 7 angles of incidence $\left(0^{\circ} \leq \alpha \leq 90^{\circ}\right)$. The velocity streamlines and vorticity contours for $\alpha=30^{\circ}$ are shown in figure 13 at four time steps of approximately one vortex shedding period $\left(T_{\mathrm{vs}}=5.85 \mathrm{~s}\right.$ for $\left.\alpha=30^{\circ}\right)$. At the top and bottom cylinder of the group (cylinders 33 and 8 in figure 13), shear layers are shed from the group while mass flow calculations indicate that about $10 \%$ of the incident flow is forced through the group. This amount was determined by comparing the incident mass flow at the inlet of the domain $\dot{m}_{\text {in }}$ (figure 14) with the mass flow deflected around the group $\dot{m}_{\text {def }}$. The latter is calculated as the mass flow passing through virtual lines drawn orthogonal to the inlet of the domain and tangent to the outermost cylinders of the group (e.g. silos 8 and 33 for $\alpha=30^{\circ}$ ), as shown in figure 14 . The flow in the interstitial spaces emerges at the lee side of the group and will be discussed in detail in paragraph 4.3. 
These emerging flows join up and form several local recirculation structures in the wake, that coalesce as they are carried downstream, eventually creating a single large scale vortex street in the wake of the entire group. Similarly as for the single cylinder case, the periodicity in this vortex street is depicted by the Strouhal number, summarized in table 2 for all angles of incidence and calculated with the characteristic length $L=D_{\mathrm{g}}$. The formation of large scale recirculation zones, made up from shedding vortices of separate cylinders, is also found in tube bundles.

[Fig. 13 about here.]

[Fig. 14 about here.]

[Table 2 about here.]

Figure 15 shows the instantaneous flow pattern for different angles of incidence. For smaller angles of incidence $\left(\alpha=0^{\circ}\right.$ and $15^{\circ}$, figures $15 \mathrm{a}$ and $\left.15 \mathrm{~b}\right)$, the emerging interstitial flows on the upper side of the group (cylinders 33 to 40) are joined up and dragged downstream without forming local recirculation zones, due to the proximity of the separated shear layer. In a similar way, no recirculation zones can be formed on the lower side of the group (cylinders 8, $16,24,32$ and 40) for the higher angles of incidence $\left(\alpha=75^{\circ}\right.$ and $90^{\circ}$, figures $15 \mathrm{e}$ and $15 \mathrm{f})$. For intermediate angles of incidence $\left(\alpha=30^{\circ}\right.$ to $60^{\circ}$, figures $15 \mathrm{c}$ and $15 \mathrm{~d})$, the free space between the shear layer and the cylinder group is larger and local recirculation zones can be formed on both downstream sides of the group, as explained for $\alpha=30^{\circ}$.

[Fig. 15 about here.] 
The asymmetry in the number of local recirculation zones formed on the downstream sides of the group is related to the angle of incidence $\alpha$ and the side ratio $L / B=1.6$ of the group and results in asymmetry in the vortex street in the wake of the group, as shown in figure 16. It should be mentioned that in the present $2 \mathrm{D}$ simulations, the vortex street is artificially preserved, while in $3 \mathrm{D}$ simulations and experiments, the vortex street would break up more quickly. This flaw in the $2 \mathrm{D}$ simulation results does not influence the explanation of the ovalling vibrations of the silos, since these vibrations are not induced by vortex shedding around the group as a whole, as will be discussed further on.

[Fig. 16 about here.]

The flow pattern around the cylinder group is clearly affected by the geometry of the group arrangement. Kareem et al. (1998) found a similar behaviour for two closely spaced cylinders in tandem arrangement: the separated shear layers interact and roll up to form one large vortex, approaching the behaviour of a single bluff body. It would therefore be interesting to compare the flow around the silo group with the flow around bluff body rectangular cylinders at the same Reynolds number. However, no experiments are available in the literature for the flow around a rectangular cylinder at high Reynolds numbers Re $\approx 10^{7}$. The result of an experiment of Knisely (1990) on a rectangular cylinder $(L / B=1.67)$ for different angles of incidence $\left(0^{\circ}<\alpha<90^{\circ}\right)$ at $1.2 \times 10^{4} \leq \operatorname{Re} \leq 2.4 \times 10^{4}$ is compared with the present simulation results in figure 17 .

[Fig. 17 about here.] 
Apart from a slightly different side ratio for the present simulations $(L / B=$ 1.6), significantly lower Strouhal numbers in the experiments of Knisely (1990) are mainly due to the large difference in Reynolds number. Although comparison of both data sets might be futile, two interesting discrepancies should be mentioned. First, data on the effects of rounded corners of the rectangular cylinders are somewhat limited and scattered but the general tendency for the Strouhal number is to increase with increasing rounding radius (Knisely, 1990). Second, the most pronounced difference between a bluff rectangular cylinder and the present cylinder group is the permeability of the latter. For a rectangular cylinder, a sudden fall in the Strouhal number is observed for very small or high angles of incidence and is interpreted as an indicator of the reattachment of the separated shear layer (Knisely, 1990). For the permeable cylinder group, the emerging interstitial flows at the lee side of the group prevent the shear layer from reattaching, explaining the absence of a sharp decrease in Strouhal number for very small or high angles of incidence. The decrease of the Strouhal number for intermediate angles of incidence $\left(\alpha=30^{\circ}\right.$ to $60^{\circ}$ ) in the present simulations is due to the lower vortex shedding frequency for these angles. When the projected width of the cylinder group $\left(D_{\mathrm{g}}\right)$ is increased, the distance between the free shear layers increases, resulting in larger shedded vortices and reduced shedding frequencies.

\subsection{Flow in the interstitial spaces of the cylinder group}

Until now, the flow pattern around the cylinder group as a whole has been discussed without consideration of the flow in the interstitial spaces between the cylinders. The internal part of the cylinder group resembles the geometry 
of tube bundles (e.g. heat exchangers) but the physical behaviour of the flow within the interstitial spaces of the silo group is fundamentally different. Two simple but important explanations can be given. Firstly, in tube bundles, the entire fluid flow is forced through the group to maximize the exchange of heat while, in the case of the cylinder group, the major part of the fluid flow is deflected around the group and approximately only $10 \%$ of the fluid flow passes through the interstitial spaces. Secondly, the pitch between cylinders is generally much larger in tube bundles than in this closely spaced silo group. Nevertheless, a short account of the differences and similarities is given in the following.

Tube arrays are typically divided in two categories (Zdravkovich, 2003): the inline category where cylinders are arranged in square or rectangle arrays and the interstitial flow is typically straight through the array gaps (Zukauskas et al., 1988) and the staggered category where cylinders are arranged in rotated square or triangle arrays and the flow is forced along wavy paths. For the latter, the near-wakes are cusped in shape, narrowed in width, and connected to enlarged stagnation regions on the upstream side of the tubes. Depending on the angle of incidence, the present silo group could be classified in either category based on geometry: the in-line, square configuration applies to the cases with $\alpha=0^{\circ}$ and $90^{\circ}$ while for all other angles of incidence the staggered, rotated square arrangement would be applicable. However, only a few similarities in the interstital flow patterns, shown in figure 18, are found. The straight flow patterns for $\alpha=0^{\circ}$ (figure 18a) and $90^{\circ}$ are not detected. For in-line tube bundles, the presence of the subsequent row prevents the transitional eddies to form and roll-up and the eddies are carried away between the tubes by the jet-like interstitial flow. If the bodies are too closely packed, 
these eddies partially or completely disappear in the distorted flow (Hunt and Eames, 2002). Instead, the interstitial flow is not separated from the cylinder wall and follows a wavy path through the array, deflecting the flow up- and downward to the sides of the group. This path emerges in the upper or lower shear layer flow respectively and corresponds to the shortest path from the high pressures at the upstream side of the group to the lower pressures at the lee side of the group. For other angles of incidence, the interstitial flow resembles the wavy interstitial flow pattern of staggered tube bundles (Zdravkovich, 2003). For $\alpha=30^{\circ}$ (figure 18c), a regular wavy pattern between the cylinders with small eddies in the interstitial spaces is observed. For $\alpha=15^{\circ}$ (figure 18b), such regular wavy pattern is not observed at some points in the array: the interstitial flow separates from the surface of the cylinder and reattaches before leaving the same interstitial space. For $\alpha=60^{\circ}$ (figure 18d), this effect is even more pronounced and the separating interstitial flows result in local vortex shedding in the interstitial spaces at arbitrary points in the array. The higher frequency peaks for these particular angles of incidence are odd and are due to a fluctuating separation point on the cylinder surface at these locations in the group.

[Fig. 18 about here.]

The presence of these small recirculation zones and vortex shedding patterns may be directly related to the $2 \mathrm{D}$ character of the simulations. Mittal and Balachandar (1995) mention simultaneous 2D and 3D simulations of the flow past circular cylinders for a large range of Reynolds numbers $\left(20<\operatorname{Re}<10^{6}\right)$, concluding that some secondary vortices in $2 \mathrm{D}$ simulations are absent in $3 \mathrm{D}$ simulations where spanwise velocities are allowed. However, flow visualization for tube bundles revealed the formation of eddies and possibly vortex shedding 
in the interstitial spaces of tube arrays as well (Zdravkovich, 2003; Price et al., 1995).

The variation of local velocities in the interstitial flow is closely related to the dissipation and generation of turbulence in the array. In several experiments on tube arrays (Zdravkovich, 2003; Moulinec et al., 2004), a quick decrease of ambient turbulence intensity after the first row was observed (order of magnitude $20 \%$ intensity reduced to $2 \%$ ) while turbulence was built up in subsequent rows of the array until the rate of turbulence generation is balanced by the turbulence dissipation (Zdravkovich, 2003). Experiments have also shown that the tighter a bundle gets, the more rapidly the interstitial flow becomes turbulized and a zone where the flow stabilizes extends to the last row of the array (Zukauskas et al., 1988). Contour lines of the instantaneous turbulence intensity for the present closely packed group of cylinders (figure 19) show similar behaviour with a swift introduction of large turbulence intensities (mean turbulence intensity values as high as 15 to $20 \%$ ) after the first row of cylinders at the upper and lower edges of the rectangular array, where the array is 'shortest' for the flow to pass.

[Fig. 19 about here.]

For all angles of incidence $\alpha$, multiple peaks in the frequency spectrum of the pressure are observed through the array as shown in figure 20 for four arbitrary points in and around the cylinder group (points A, B, C and D in figure 2). It can be seen that the frequency spectrum of the interstitial flow is more or less independent of the location. For each angle of incidence, the lowest peak in the spectrum is identified with the Strouhal frequency (table 2). The higher frequency peaks are multiples of the Strouhal frequency; for $\alpha=0^{\circ}$ (figure 
20a) and $90^{\circ}$ the first two peaks are dominant, while for all other angles (e.g. $\alpha=30^{\circ}$, figure 20c), only the first peak is clearly pronounced. Note that at the lee side of the group, no higher frequency peaks are observed (e.g. in point D) since no small vortex shedding exists in separate wakes of these cylinders. For two angles of incidence, higher frequency peaks are found as well: for $\alpha=15^{\circ}$ (figure 20b) small peaks are distinguished at $6 \mathrm{~Hz}$ but, more significantly, for $\alpha=60^{\circ}$ (figure 20d) important frequency peaks are detected at $2 \mathrm{~Hz}$. These frequency peaks are explained by the deviations of the regular wavy pattern for these angles of incidence (figures $18 \mathrm{~b}$ and 18d).

[Fig. 20 about here.]

Experiments by Polak and Weaver (1995) have shown that, as flow develops in the interstitial spaces of tube bundles, local flow velocity changes in every row and triggers vortex shedding at discrete, row dependent frequencies. A few tube rows downstream the flow stabilizes, turbulence reaches a maximum and measured results become row independent. This was for example found in experiments by Price et al. (1987) where up to three frequency peaks, interrelated with ratios 1:2:3, could be identified in the upstream rows of a staggered tube array while only one frequency peak remained in the downstream rows. Experiments by Weaver et al. (1993) yield similar results with two narrow band peaks at $3.7 \mathrm{~Hz}$ and $5.8 \mathrm{~Hz}$ until the third row of a staggered tube array and only the lower frequency peak persisting in subsequent rows. In small pitch ratio tube bundles, these flow fluctuations become rapidly distorted and diffused and coherent vortical structures cannot be detected as deeply into the array (Polak and Weaver, 1995). Hence, in the present simulations of the closely spaced silo group, different frequencies are expected depending on local flow conditions and geometry. This is demonstrated by the different narrow 
band frequency spectra of the present data with flow orientation (figure 20). However, the narrow band frequency spectra do not vary significantly with progress through the array, although the point furthest downstream (point D, figure 20) does show magnitude differences with the upstream perturbations (points A, B and C).

\subsection{Dynamic wind pressure loads on the cylinder surfaces of the group}

To clarify the cause of the observed ovalling oscillations at the lee side corner silos of the present silo group, pressure distributions on the walls of the cylinders are considered. For design purposes, both time averaged pressures and fluctuating pressures should be considered, the first as an indication for the static deflection and the latter for the dynamic excitation of the silos. Since static, time averaged pressures are uncapable of triggering oscillations, only the dynamic, fluctuating wind pressures on the silo surfaces should be examined to clarify the observed ovalling vibrations in the 8 by 5 silo group.

Fluctuating pressure coefficients along the circumference of a cylinder and at each time step are determined as follows:

$$
C_{\mathrm{p}}^{\prime}(\theta, t)=C_{\mathrm{p}}(\theta, t)-\bar{C}_{\mathrm{p}}(\theta)
$$

The fluctuating pressure coefficient $C_{\mathrm{p}}^{\prime}(\theta, t)$ is harmonically decomposed at every time step $t$ into a series of cosine functions with circumferential wavenumber $n$, corresponding to the mode shapes of the axisymmetric structure:

$$
C_{\mathrm{p}}^{\prime}(\theta, t)=\sum_{n=0}^{\infty} C_{\mathrm{p}}^{\prime n}(t) \cos \left(n \theta+\phi_{n}\right)
$$


To assess the dynamic response of the cylinders, the time history of the amplitudes $C_{\mathrm{p}}^{\prime n}(t)$ is subsequently transformed to the frequency domain by means of a FFT algorithm, yielding $C_{\mathrm{p}}^{\prime n}(f)$. Wavenumber-frequency spectra of these amplitudes $C_{\mathrm{p}}^{\prime n}(f)$ for an angle of incidence $\alpha=30^{\circ}$ are shown for all cylinders of the group in figure 21.In these spectra, the colour of the band peaks is a measure for the excitation level of the dynamic pressure loads on the cylinder surfaces while the width is a measure for their steadiness. Thus, a dark narrow band peak close to a structural natural frequency is very likely to excite resonance.

[Fig. 21 about here.]

[Fig. 22 about here.]

It is observed that the contribution in the spectrum of the pressure fluctuations on the cylinder surfaces increases when moving downstream to the lee side of the group (e.g. silo 1 and 10 vs. silos 31 and 40 in figure 21). At the same time, it is clear that the frequency contribution rapidly decreases as the wavenumber $n$ and the frequency $f$ increase (e.g. in the spectra for silo 1 and 33 in figure 21), corresponding to measured wind spectra with typically low frequency components. One could hence already conclude that only structural mode shapes with low natural frequencies $f_{\text {eig }}$ and a limited circumferential wavenumber $n$ can possibly be excited by the dynamic wind pressures (figure 3).

Looking in more detail at the wavenumber-frequency spectra $C_{\mathrm{p}}^{\prime n}(f)$ in figure 21 , it is observed that the spectra at the upstream part of the group show no periodicities other than the low frequency contributions related to the large vortex shedding in the wake of the group $\left(f_{\mathrm{vs}}\right.$, table 2$)$. However, following the 
wind motion towards the lee side of the group, irregularities appear, growing onwards to cylinder 40. In this part of the group, e.g. for silo 30, two distinctive narrow band higher frequency peaks can be observed between $3 \mathrm{~Hz}$ and $4 \mathrm{~Hz}$ and also at approximately $6.5 \mathrm{~Hz}$. Taking into account the corresponding circumferential wavenumbers $n$ on the vertical axis, it is concluded that the third and fourth circumferential eigenmodes $(1,3)$ and $(1,4)$ of the silos (figure 3 ), both at eigenfrequencies of $f_{\text {eig }}=3.93 \mathrm{~Hz}$ would probably be excited by the dynamic wind pressures. The excitation of higher ovalling eigenmodes is also possible but vibration amplitudes will be smaller since their eigenfrequencies $f_{\text {eig }}$ are just below or above the frequency peaks at $6.5 \mathrm{~Hz}$. In the case of $\alpha=30^{\circ}$, the ovalling eigenmodes are not only excited at the lee side of the group, but also further upstream: e.g. for silos 6 to 8 the darker narrow band peaks in the spectrum at $3 \mathrm{~Hz}$ to $4 \mathrm{~Hz}$ for $n=3$ and $n=4$ are already observed.

In figure 22, wavenumber-frequency spectra are shown for the corner silos of the group (silo 1, 8, 33 and 40) for different angles of incidence $\alpha$ to investigate if the same tendencies are found as for $\alpha=30^{\circ}$. The wavenumber-frequency spectra for $\alpha=30^{\circ}$ and $\alpha=45^{\circ}$ are very similar. In the spectrum for silo 40 at $\alpha=45^{\circ}$, the peak at approximately $4 \mathrm{~Hz}$ is even more pronounced than for $\alpha=30^{\circ}$. As mentioned previously, for $\alpha=30^{\circ}$ the excitation of ovalling eigenmodes exists further upstream (e.g. already for silo 8) than for other angles of incidence. The results for $\alpha=15^{\circ}$ and $\alpha=60^{\circ}$ deviate somewhat from the general tendencies described until now, due to the higher frequency peaks at respectively $6 \mathrm{~Hz}$ and $2 \mathrm{~Hz}$. However, these irregular peaks are likely inconsequential for the ovalling vibrations observed in this case, due to a significant mismatch between the excitation frequency and the structural natural 
frequencies. Besides these deviant peaks, the results for $\alpha=15^{\circ}$ and $\alpha=60^{\circ}$ agree qualitatively well with the results for $\alpha=30^{\circ}$ and $\alpha=45^{\circ}$. Finally, also wavenumber-frequency spectra are shown for $\alpha=0^{\circ}$ and $\alpha=90^{\circ}$. Pressure distributions on upstream cylinder surfaces are very similar as for other angles of incidence. Note that for $\alpha=0^{\circ}$ the upstream corners of the group are at silos 1 and 33 while for $\alpha=90^{\circ}$ the upstream corner silos are 1 and 8, explaining the 'switching' of wavenumber-frequency spectra of these two cylinders. At the lee side of the group, similar results are found as for other angles of incidence, e.g. silo 40.

[Fig. 23 about here.]

\section{Ovalling vibrations in the 8 by 5 silo group}

From the above results, the existence of ovalling vibrations at the lee side corner silos of a silo group can be demonstrated. Considering the dynamic excitation of the silos, ovalling vibrations will preferentially be induced at the lee side of the group while the third and fourth eigenmode of the silos are most likely to be excited. This corresponds with the observed pattern of ovalling vibrations with three and four circumferential wavelengths during the 2002 storm in Antwerp. Depending on the orientation of the group, these ovalling oscillations may not only exist at the lee side of the group but also further upstream (e.g. silos 7 and 8 for $\alpha=30^{\circ}$ ).

Although the existence of these ovalling vibrations was demonstrated, the physical phenomenon causing these vibrations has not yet been explained. Many researchers have detected periodic forces on cylinders in arrays when 
subject to cross flow (Price et al., 1987). It is generally accepted that there are three distinct mechanisms leading to vibrations in a tube array (Price et al., 1987; Weaver et al., 1993; Zukauskas et al., 1988):

(1) Forces in tube arrays arise due to turbulent fluctuations of the flow pressure. Turbulent buffeting fluctuations of flow velocity, either initiated upstream or induced within the array itself, are converted into pressure fluctuations on the cylinder surfaces (Price et al., 1987).

(2) Forces are induced by fluidelastic instability (FEI). These self-excited forces arise when the tubes move out of their equilibrium position and tube motion and fluid flow start to interact. The fluidelastic forces are proportional to tube displacement at the onset of instability and are superimposed by a second type of forces, proportional to tube vibration velocities (Schroder and Gelbe, 1999).

(3) Forces in cylinder arrays can be due to periodic vortex shedding. While there used to be debate in the literature whether there was sufficient space for vortices to develop in interstitial spaces or not (Price et al., 1995), it is now known that coherent vortex shedding exists in tube arrays but the coherent vorticity has no room to produce a vortex street. Vortex shedding and FEI generally coexist as separate and independent phenomena in tube arrays but, in certain array geometries in water flows, coupling may occur and it becomes difficult to separate the two (Weaver et al., 2000).

It should be noted that typical vibration phenomena in tube arrays reported in the literature, where the entire 'rigid' tube moves out of its equilibrium 
position, are very different from the ovalling vibrations in this study, where only the tube wall is vibrating. While the prevailing physical phenomena are expected to be similar, the specific excitation mechanisms causing large amplitude vibrations of heat exchanger tubes and ovalling oscillations of silo structures are almost certainly different. In the following it is investigated which of these physical phenomena can cause the observed ovalling vibrations.

Previous research has convincingly shown that conventional vortex shedding cannot excite ovalling oscillations of cylindrical shells in cross flow since ovalling of a single cylinder also occurs when a long splitter plate in the wake suppresses periodic vortex shedding(Païdoussis et al., 1988). This is confirmed by later experimental research (Laneville and Mazouzi, 1995) as well as in the present simulations since the structural natural frequencies $\left(f_{\text {eig }}\right.$, figure 3$)$ do not match the global vortex shedding frequency in the wake of the entire group $\left(f_{\mathrm{vs}}\right.$ in table 2$)$. No higher frequencies due to vortex shedding in individual silo wakes of the type typically found in heat exchangers have been detected either (e.g. point D in figure 20). Since no coupling of fluid and structural motions is included, FEI is not considered and can therefore not be ruled out entirely by this study. Hence, only turbulent buffeting remains which could cause the narrow band wake phenomena. The numerical analysis suggests that there is significant narrow band energy in the flow at frequencies very close to the structural frequencies that could explain the observed silo vibrations.

From an engineering point of view, alternative arrangements of the cylinders could be considered to reduce the risk of structural damage. This is however a difficult task, since wind directions change and different silos will hence be located at the lee side of the group for different wind directions. A more economical solution to prevent ovalling vibrations is to stiffen the silo structures, 
e.g. by thickening the walls of the silos, to shift the structural natural frequencies of the structures to higher values. However, if flow-excited resonance with narrow band wake phenomena, as discussed above, is the source of the problem, one then has to prevent that the modified, higher natural frequencies match a higher frequency in the flow (e.g. $6.5 \mathrm{~Hz}$ for silos 29 , 30, etc. in figure 21).

\section{Conclusions}

In order to elucidate the occurence of ovalling vibrations on the empty lee side corner silos of a 8 by 5 group in the port of Antwerp, the post-critical flow for 7 angles of incidence $\alpha$ between $0^{\circ}$ and $90^{\circ}$ around this closely spaced cylinder group was modelled using 2D URANS simulations. For the validation of the computational model and its spatial and temporal grid refinement, simulations for a single cylinder were performed and qualitative validation was done by comparing the present results with the flow around bluff rectangular cylinders and the flow through tube bundles.

The simulations have shown that the silo group can be treated neither as a tube array nor as a solid bluff body. While the group configuration drastically changes the flow regime around the group, similar to bluff rectangular cylinders, the permeability allows about $10 \%$ of the mass flow to pass through the group. Compared to heat exchangers where the entire mass flow is forced through the array, it is almost certain that the excitation mechanisms causing tube vibrations in heat exchangers and the present silo wall oscillations will be different. The interstitial flows in the silo group follow wavy patterns through the array for all angles of incidence, from high upstream pressures to lower 
pressures at the lee side of the group. Some irregularities at arbitrary locations in the array for $\alpha=15^{\circ}$ and for $\alpha=60^{\circ}$ are attributed to the 2D character of the simulations where spanwise velocities are suppressed. The interstitial flows emerge at the lee side of the group and form local recirculation zones which coalesce to form a large vortex street in the wake of the group.

The observed ovalling vibrations in the silo group are investigated by linking the dynamic wind pressures on the silo surfaces to the dynamic properties of the structures. For all orientations of the group $\left(0^{\circ}<\alpha<90^{\circ}\right)$, strong narrow band frequency peaks are identified in the turbulent pressure coefficient spectra of the silos near the lee side corners of the group that match the structural natural frequencies of the third and fourth ovalling mode shape of the silos. This match indicates a forced, resonant response which corresponds with the observed pattern of ovalling vibrations with three and four circumferential wavelenghts near the lee side corners of the silo group during the 2002 storm in Antwerp. While the precise physical excitation mechanism is not yet fully understood, the simulations confirm that discrete vortex shedding (in the wake of the entire group or by vortices in the individual silo wakes of the type found in heat exchangers) can be excluded. Since no coupling of fluid and structural motions was included in this study, FEI could not be considered. Hence, only turbulent buffeting remains which could very well give rise to the narrow band wake phenomena causing the ovalling silo wall vibrations.

\section{Acknowledgements}

The research in this paper has been performed within the frame of the FWO project G.0275.08 "Efficient analysis of fluid-structure interaction problems in 
structural dynamics". The fourth author is a post-doc fellow of the Research Foundation Flanders (FWO). The support of FWO is gratefully acknowledged. Furthermore, the help of professor A. Roshko with the experimental data of Shih et al. (1993) and the constructive remarks of the editor regarding vibration phenomena in tube bundles were greatly appreciated. 


\section{References}

Ansys, 2009. Theory Guide, ANSYS FLUENT Release 12.0. Ansys Inc.

Behr, M., Hastreiter, D., Mittal, S., Tezduyar, T.E., 1995. Incompressible flow past a circular cylinder: dependence of the computed flow field on the location of the lateral boundaries. Computer Methods in Applied Mechanics and Engineering 123, 309-316.

BIN, 2005. NBN EN 1991-1-4:2005 Eurocode 1: Actions on structures - Part 1-4: General actions - Wind actions. Belgisch Instituut voor Normalisatie.

Catalano, P., Wang, M., Iaccarino, G., Moin, P., 2003. Numerical simulation of the flow around a circular cylinder at high Reynolds numbers. International Journal of Heat and Fluid Flow 24, 463-469.

Celik, I., Shaffer, F.D., 1995. Long time-averaged solutions of turbulent flow past a circular cylinder. Journal of Wind Engineering and Industrial Aerodynamics 56, 185-212.

Dooms, D., Degrande, G., De Roeck, G., Reynders, E., 2006. Finite element modelling of a silo based on experimental modal analysis. Engineering Structures $28,532-542$.

ESDU, 1985. Data Item No. 85020: Characteristics of atmospheric turbulence near the ground. Part II: single point data for strong winds (neutral atmosphere). Engineering Science Data Unit, London, UK.

Holloway, D.S., Walters, D.K., Leylek, J.H., 2004. Prediction of unsteady, separated boundary layer over a blunt body for laminar, turbulent, and transitional flow. International Journal for Numerical Methods in Fluids $45,1291-1315$.

Hunt, J.C.R., Eames, I., 2002. The disappearance of laminar and turbulent wakes in complex flows. Journal of Fluid Mechanics 457, 111-132. 
Issa, R.I., 1986. Solutions of the implicitly discetised fluid flow equations by operator-splitting. Journal of Computational Physics 62, 40-65.

James, W.D., Paris, S.W., Malcolm, G.N., 1980. Study of viscous crossflow effects on circular cylinders at high Reynolds numbers. AIAA Journal 18, 1066-1072.

Kareem, A., Kijewski, T., Lu, P.C., 1998. Investigation of interference effects for a group of finite cylinders. Journal of Wind Engineering and Industrial Aerodynamics 77-78, 503-520.

Knisely, C.W., 1990. Strouhal numbers of rectangular cylinders at incidence: a review and new data. Journal of Fluids and Structures 4, 371-393.

Laneville, A., Mazouzi, A., 1995. Ovalling oscillations of cantilevered cylindrical shells in cross-flow: New experimental data. Journal of Fluids and Structures 9, 729-745.

Menter, F.R., 1994. Two-Equation Eddy-Viscosity Turbulence Models for Engineering Applications. AIAA Journal 32, 1598-1605.

Mittal, R., Balachandar, S., 1995. Effect of three-dimensionality on the lift and drag of nominally two-dimensional cylinders. Physics of Fluids 7, 18411865.

Moulinec, C., Pourquié, M.J.B.M., Boersma, B.J., Buchal, T., Nieuwstadt, F.T.M., 2004. Direct numerical simulation on a cartesian mesh of the flow through a tube bundle. International Journal of Computational Fluid Dynamics 18, 1-14.

Ong, M.C., Utnes, T., Holmedal, L.E., Myrhaug, D., Pettersen, B., 2009. Numerical simulation of flow around a smooth circular cylinder at very high Reynolds numbers. Marine Structures 22, 142-153.

Païdoussis, M.P., Price, S.J., Ang, S.-Y., 1988. Ovalling oscillations of cylindrical shells in cross flow: A review and some new results. Journal of Fluids 
and Structures 2, 95-112.

Païdoussis, M.P., Price, S.J., Suen, H.C., 1982. Ovalling oscillations of cantilevered and clamped-clamped cylindrical-shells in cross flow: An experimental-study. Journal of Sound and Vibration 83, 533-553.

Patankar, S.V., Spalding, D.B., 1972. A calculation procedure for heat, mass and momentum transfer in three-dimensional parabolic flows. International Journal of Heat and Mass Transfer 15, 1787-1806.

Polak, D.R., Weaver, D.S., 1995. Vortex shedding in normal triangular tube arrays. Journal of Fluids and Structures 9, 1-17.

Price, S.J., Païdoussis, M.P., Macdonald, R., Mark, B., 1987. The flow-induced vibration of a single flexible cylinder in a rotated square array of rigid cylinders with a pitch-to-diameter ratio of 2.12. Journal of Fluids and Structures $1,359-378$.

Price, S.J., Païdoussis, M.P., Mark, B., 1995. Flow visualization of the interstitial cross-flow through parallel triangular and rotated square arrays of cylinders. Journal of Sound and Vibration 181, 85-98.

Saghafian, M., Stansby, P.K., Saidi, M.S., Apsley, D.D., 2003. Simulation of turbulent flows around a circular cylinder using nonlinear eddy-viscosity modelling: steady and oscillatory ambient flows. Journal of Fluids and Structures 17, 1213-1236.

Sak, C., Liu, R., Ting, D.S.-K., Rankin, R.W., 2007. The role of turbulence length scale and turbulence intensity on forced convection from a heated horizontal circular cylinder. Experimental Thermal and Fluid Science 31, 279-289.

Schewe, G., 1983. On the force-fluctuations acting on a circular-cylinder in cross-flow from subcritical up to transcritical Reynolds-numbers. Journal of Fluid Mechanics 133, 265-285. 
Schroder, K., Gelbe, H., 1999. New design recommendations for fluidelastic instability in heat exchanger tube bundles. Journal of Fluids and Structures $13,361-379$.

Shih, W.C.L., Wang, C., Coles, D., Roshko, A., 1993. Experiments on flow past rough circular cylinders at large Reynolds numbers. Journal of Wind Engineering and Industrial Aerodynamics 49, 351-368.

Travin, A., Shur, M., Strelets, M., Spalart, P., 2000. Detached-eddy simulations past a circular cylinder. Flow, Turbulence and Combustion 63, $293-313$.

Versteeg, H.K., Malalasekara, W., 2007. An Introduction to Computational Fluid Mechanics: The Finite Volume Method. Pearson Education Limited, Essex, England. second edition.

Weaver, D.S., Lian, H.Y., Huang, X.Y., 1993. Vortex shedding in rotated square arrays. Journal of Fluids and Structures 7, 107-121.

Weaver, D.S., Ziada, S., Au-Yang, M.K., Chen, S.S., 2000. Flow-induced vibrations in power and process plant componentsprogress and prospects. Journal of Pressure Vessel Technology 122, 339-348.

Younis, B.A., Przulj, V.P., 2006. Computation of turbulent vortex shedding. Computational Mechanics 37, 408-425.

Zan, S.J., 2008. Experiments on circular cylinders in crossflow at Reynolds numbers up to 7 million. Journal of Wind Engineering and Industrial Aerodynamics 96, 880-886.

Zdravkovich, M.M., 1997. Flow Around Circular Cylinders, Volume 1: Fundamentals. Oxford University Press, Oxford, England.

Zdravkovich, M.M., 2003. Flow Around Circular Cylinders, Volume 2: Applications. Oxford University Press, Oxford, England.

Zukauskas, A., Ulinskas, R., Katinas, V., Karni, J., 1988. Fluid Dynamics and 
Flow-Induced Vibrations of Tube Banks. Hemisphere Publishing Corporation, New York, USA. 


\section{List of Figures}

1 Photo (a) and lateral view (b) of the silo group.

2 Plan view of the silo group with numbering of the individual silos. Normative dimensions are given as well as definitions for the angle of incidence $\alpha$ and the angle $\theta$ on the circumference of an individual cylinder.

3 Top view of the displacements of the shell sections between $15 \mathrm{~m}$ and $17.5 \mathrm{~m}$ above the bottom cone, and a $3 \mathrm{D}$ view of selected ovalling mode shapes of a single silo, (a) mode $(1,3)$ at $3.93 \mathrm{~Hz}$, (b) mode $(1,4)$ also at $3.93 \mathrm{~Hz}$, (c) mode $(1,5)$ at $5.25 \mathrm{~Hz},(\mathrm{~d})$ mode $(1,6)$ at $7.37 \mathrm{~Hz}$ and (e) mode $(1,2)$ at $7.75 \mathrm{~Hz}$ (Dooms et al., 2006).

4 Geometry of the computational domain for the single cylinder case.

5 Convergence of (a) drag coefficient $C_{\mathrm{d}}$ and (b) lift coefficient $C_{\mathrm{l}}$ for increasing spatial grid refinement in the near-wall region. Meshes with $\mathrm{AR}=12.5,25,37.5$ and 50 are located in the log-law region ( $\boldsymbol{\bullet}$ for time averaged coefficient), $\mathrm{AR}=100,200$ and 300 are located in the buffer layer $(x$ for time averaged coefficient) and $\mathrm{AR}=320$ is located in the viscous sublayer for time averaged coefficient). Lower and upper limits of the vertical intervals are respectively minimal and maximal drag and lift coefficients.

6 Computational mesh for the single cylinder case with $\mathrm{AR}=50$ and $y_{\max }^{+}=97$ (a) for the entire computational domain and details of (b) the near-wake region and (c) the near-wall region of the cylinder.

$7 \quad$ Convergence of the maximal lift coefficient $C_{1}^{\max }$ for decreasing time step. The horizontal axis is scaled inversely proportional to the time step: $0.01 / \Delta t$. Note that this figure is equivalent to figure $5 \mathrm{~b}$, since $C_{1}^{\max }=-C_{1}^{\min }$ and $C_{1}^{\text {avg }}=0$.

8 Time averaged pressure coefficient $\bar{C}_{\mathrm{p}}$ on the circumference of the cylinder with indication of the free stream velocity $v_{\infty}$, the separation angle $\theta_{\mathrm{s}}$, the time averaged base pressure coefficient $\bar{C}_{\mathrm{p}}^{\mathrm{b}}$ and the minimum time averaged pressure coefficient $\bar{C}_{\mathrm{p}}^{\mathrm{min}}$. 
9 Comparison of the present calculated $\left(y^{+}=97\right)$ maximal, minimal (dashed lines) and time averaged pressure coefficients $\bar{C}_{\mathrm{p}}(\theta)$ (solid line) for the flow around a single cylinder at $\mathrm{Re}=1.24 \times 10^{7}$ with experimental results of Zdravkovich (1997) for $0.73 \times 10^{7} \leq \operatorname{Re} \leq 3.65 \times 10^{7}$ (dark grey zone) and Shih et al. (1993) at $\operatorname{Re}=0.8 \times 10^{7}(\circ)$.

10 Computational mesh for the 8 by 5 silo group at an angle of incidence $\alpha=30^{\circ}$, shown for (a) the entire computational domain and details of $(b, c)$ the near-wake region and $(d)$ the near-wall region.

11 Time history of drag coefficients for the corner silos of the 8 by 5 silo group (silo 1 - dashed black line; silo 8 - solid black line; silo 33 - dashed grey line; silo 40 - solid grey line) with wind flow at angle of incidence $\alpha=30^{\circ}$ and for inlet turbulence intensities of (a) $\mathrm{Tu}=0.1 \%$, (b) $\mathrm{Tu}=1 \%$ and (c) $\mathrm{Tu}=10 \%$.

12 Time history of lift coefficients for the corner silos of the 8 by 5 silo group (silo 1 - dashed black line; silo 8 - solid black line; silo 33 - dashed grey line; silo 40 - solid grey line) with wind flow at angle of incidence $\alpha=30^{\circ}$ and for inlet turbulence intensities of (a) $\mathrm{Tu}=0.1 \%$, (b) $\mathrm{Tu}=1 \%$ and (c) $\mathrm{Tu}=10 \%$.

13 Velocity streamlines and vorticity contours (filled) of the flow around the 8 by 5 cylinder group for an angle of incidence $\alpha=30^{\circ}$ at (a) $t=77.0 \mathrm{~s}$, (b) $t=78.5 \mathrm{~s}$, (c) $t=80.0 \mathrm{~s}$, and (d) $t=81.5 \mathrm{~s}$.

14 Schematic representation of the incident mass flow at the inlet of the domain $\dot{m}_{\text {in }}$ and the mass flow deflected around the silo group $\dot{m}_{\text {def }}$.

15 Velocity streamlines and vorticity contours (filled) of the flow around the 8 by 5 cylinder group for an angle of incidence (a) $\alpha=0^{\circ}$ at $t=80.0 \mathrm{~s}$, (b) $\alpha=15^{\circ}$ at $t=82.5 \mathrm{~s}$, (c) $\alpha=30^{\circ}$ at $t=77.0 \mathrm{~s},(\mathrm{~d}) \alpha=60^{\circ}$ at $t=76.5 \mathrm{~s}$, (e) $\alpha=75^{\circ}$ at $t=80.5 \mathrm{~s}$, and (f) $\alpha=90^{\circ}$ at $t=83.5 \mathrm{~s}$.

16 Turbulence intensity in the flow around the 8 by 5 cylinder group for an angle of incidence (a) $\alpha=0^{\circ}$ at $t=78.5 \mathrm{~s}$ and (b) $\alpha=30^{\circ}$ at $t=77.0 \mathrm{~s}$. 
17 Comparison of Strouhal numbers for the present 8 by 5 silo group at $\operatorname{Re}=1.24 \times 10^{7}(\mathrm{o})$ and a rectangular cylinder with side ratio $L / B=1.67$ at Re between 1.2 and $2.4 \times 10^{4}(\triangle)$ (Knisely, 1990) for angles of incidence $\alpha$ between $0^{\circ}$ and $90^{\circ}$.

18 Detail of velocity streamlines and vorticity contours (filled) for the interstitial space in the 8 by 5 cylinder group for an angle of incidence (a) $\alpha=0^{\circ}$ at $t=78.5 \mathrm{~s}$, (b) $\alpha=15^{\circ}$ at $t=77.0 \mathrm{~s}$, (c) $\alpha=30^{\circ}$ at $t=79.0 \mathrm{~s}$, and (d) $\alpha=60^{\circ}$ at $t=85.0 \mathrm{~s}$.

19 Contour plot of the turbulence intensity within the cylinder array, for an angle of incidence $\alpha=30^{\circ}$ at $t=77.0 \mathrm{~s}$.

20 Frequency spectra for the pressure in points A, B, C and D (figure 2) for angles of incidence (a) $\alpha=0^{\circ}$, (b) $\alpha=15^{\circ}$, (c) $\alpha=30^{\circ}$, and (d) $\alpha=60^{\circ}$.

21 Wavenumber-frequency spectra of the amplitude $C_{\mathrm{p}}^{\prime n}(f)$ for angle of incidence $\alpha=30^{\circ}$ : upstream part of the group.

21 Wavenumber-frequency spectra of the amplitude $C_{\mathrm{p}}^{\prime n}(f)$ for angle of incidence $\alpha=30^{\circ}$ : downstream part of the group.

22 Wavenumber-frequency spectra of the amplitude $C_{\mathrm{p}}^{\prime n}(f)$ on the corner cylinders $(1,8,33$ and 40$)$ of the group for angle of incidence (a) $\alpha=0^{\circ}$, (b) $\alpha=15^{\circ}$, (c) $\alpha=30^{\circ}$, (d) $\alpha=45^{\circ}$, (e) $\alpha=60^{\circ}$ and (f) $\alpha=90^{\circ}$. 

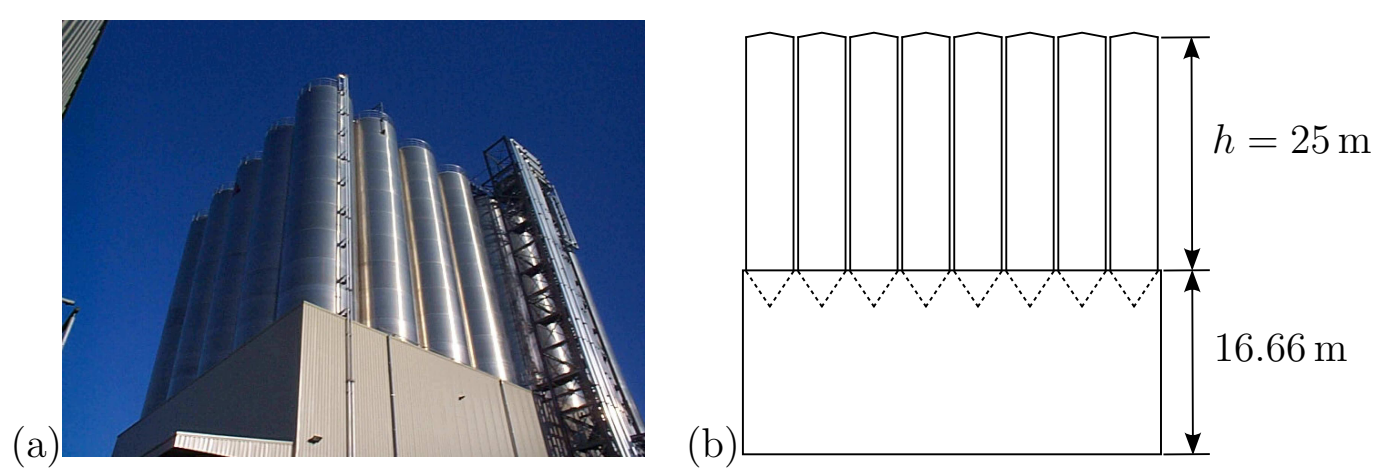

Fig. 1. Photo (a) and lateral view (b) of the silo group. 


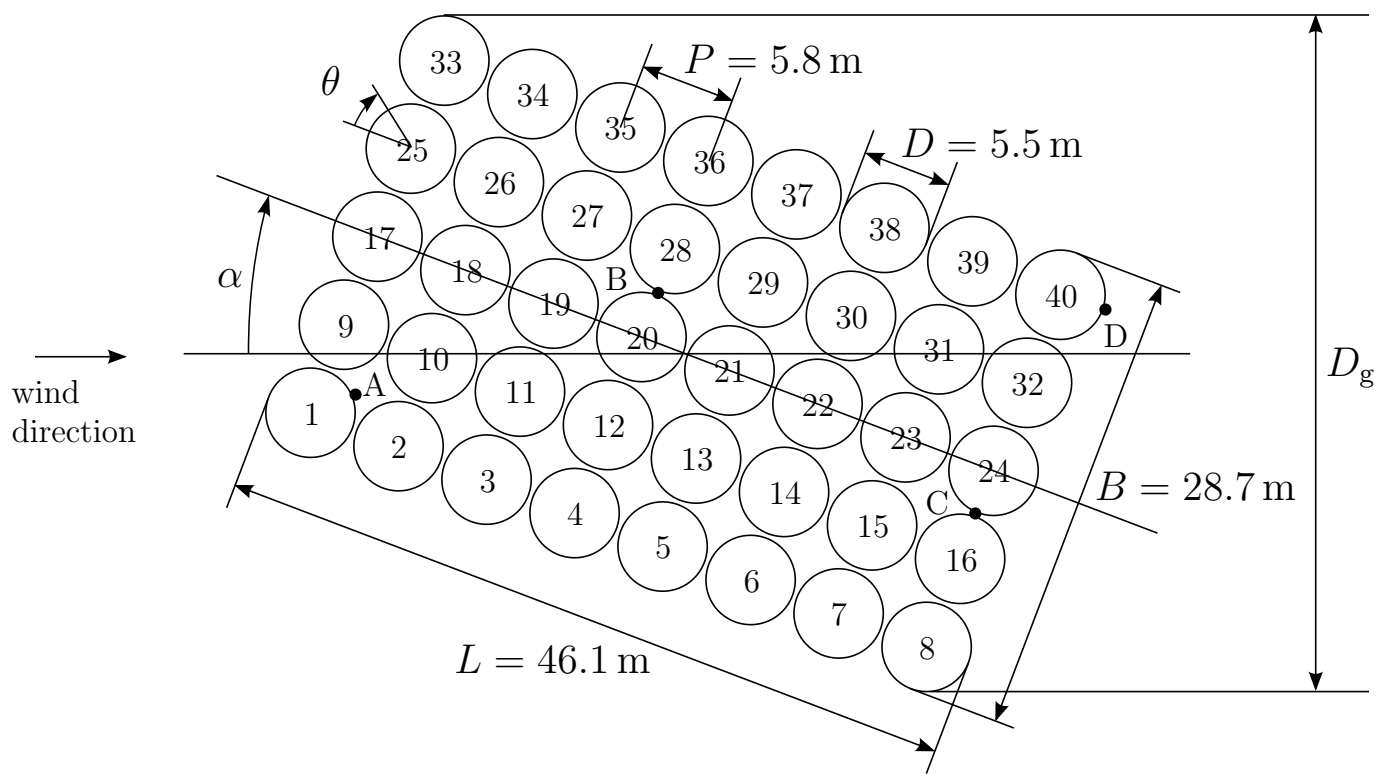

Fig. 2. Plan view of the silo group with numbering of the individual silos. Normative dimensions are given as well as definitions for the angle of incidence $\alpha$ and the angle $\theta$ on the circumference of an individual cylinder. 


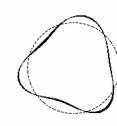

(a)

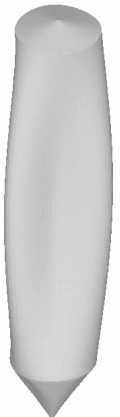

(b)<smiles>C1CCCC1</smiles>

(c)

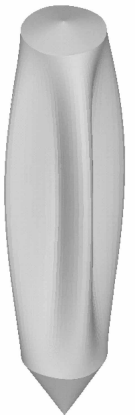<smiles>C1CC2CCC1C2</smiles>

Fig. 3. Top view of the displacements of the shell sections between $15 \mathrm{~m}$ and $17.5 \mathrm{~m}$ above the bottom cone, and a $3 \mathrm{D}$ view of selected ovalling mode shapes of a single silo, (a) mode $(1,3)$ at $3.93 \mathrm{~Hz}$, (b) mode $(1,4)$ also at $3.93 \mathrm{~Hz}$, (c) mode $(1,5)$ at $5.25 \mathrm{~Hz},(\mathrm{~d})$ mode $(1,6)$ at $7.37 \mathrm{~Hz}$ and (e) mode $(1,2)$ at $7.75 \mathrm{~Hz}$ (Dooms et al., 2006). 


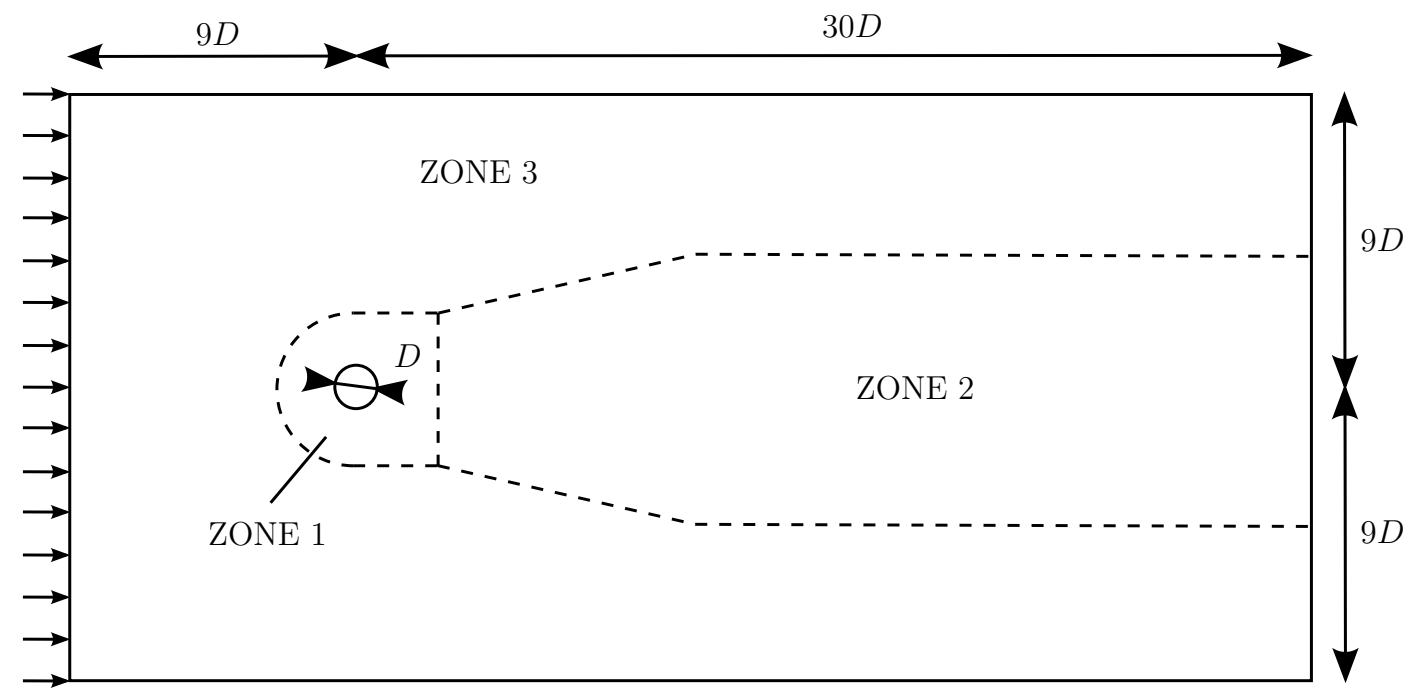

Fig. 4. Geometry of the computational domain for the single cylinder case. 


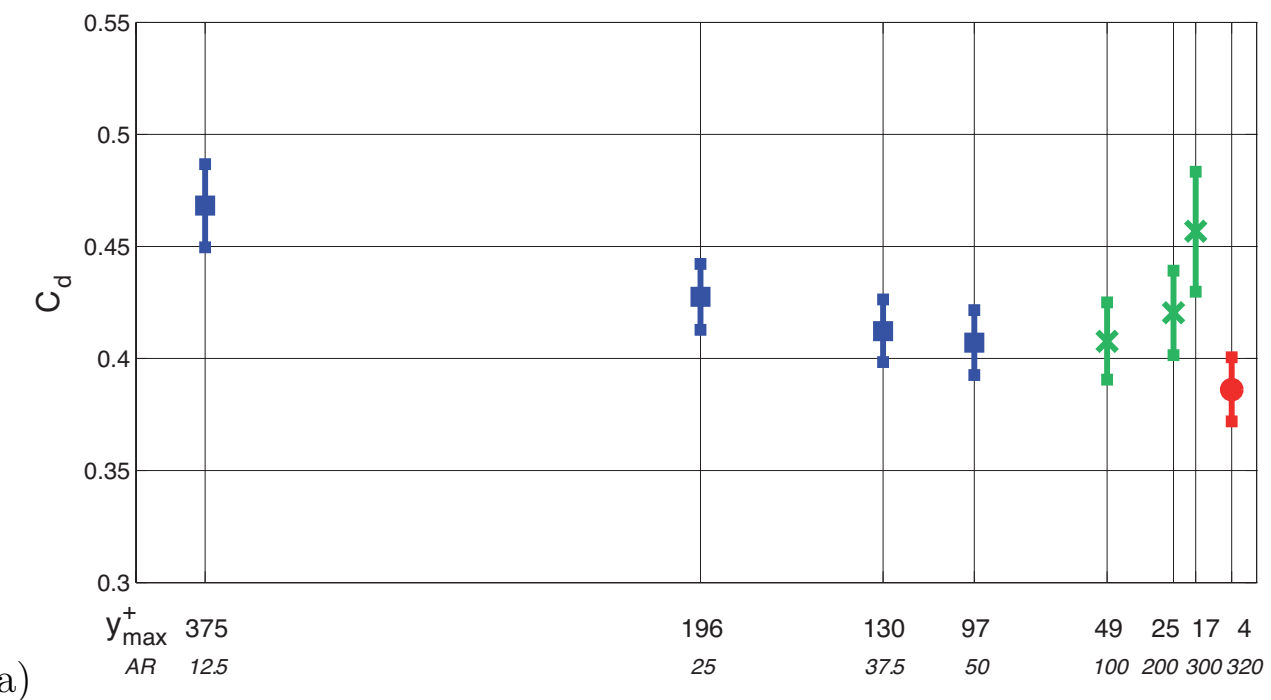

(a)

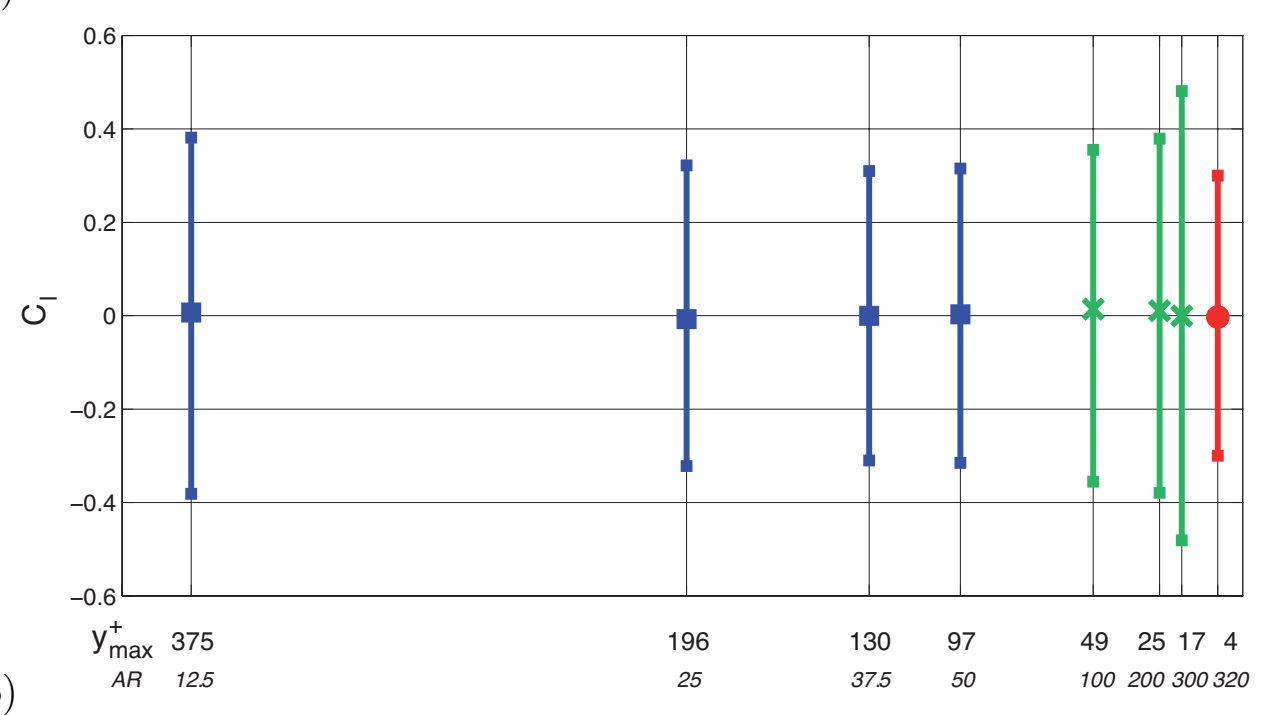

(b)

Fig. 5. Convergence of (a) drag coefficient $C_{\mathrm{d}}$ and (b) lift coefficient $C_{\mathrm{l}}$ for increasing spatial grid refinement in the near-wall region. Meshes with $\mathrm{AR}=12.5,25,37.5$ and 50 are located in the log-law region ( for time averaged coefficient), AR $=100$, 200 and 300 are located in the buffer layer $(\times$ for time averaged coefficient) and $\mathrm{AR}=320$ is located in the viscous sublayer ( $\bullet$ for time averaged coefficient). Lower and upper limits of the vertical intervals are respectively minimal and maximal drag and lift coefficients. 
(a)
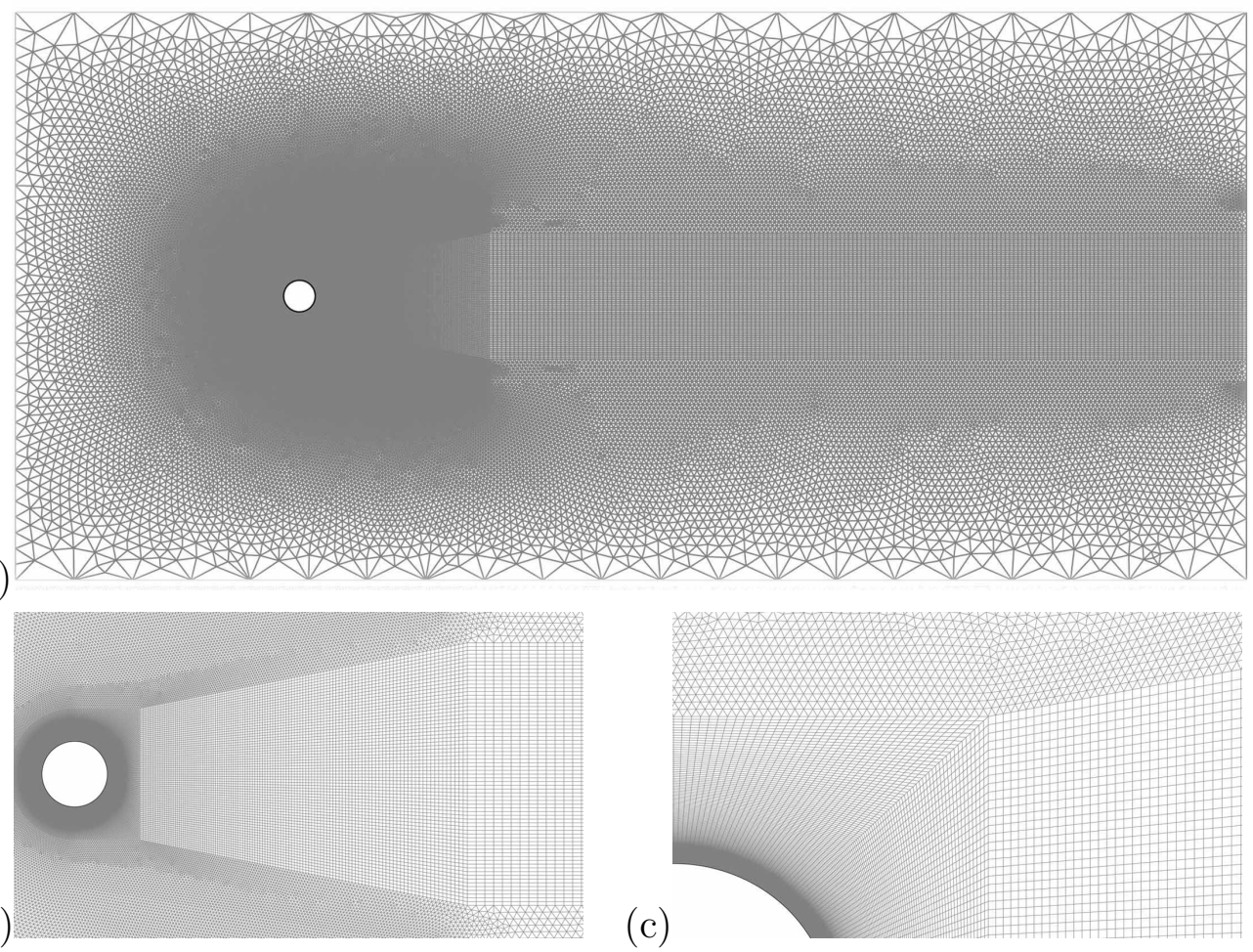

(c)

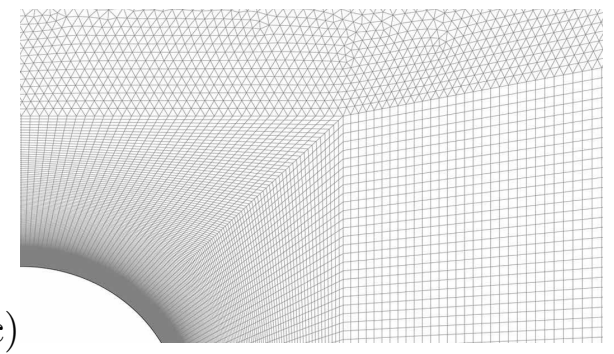

Fig. 6. Computational mesh for the single cylinder case with $\mathrm{AR}=50$ and $y_{\max }^{+}=97$ (a) for the entire computational domain and details of (b) the near-wake region and (c) the near-wall region of the cylinder. 


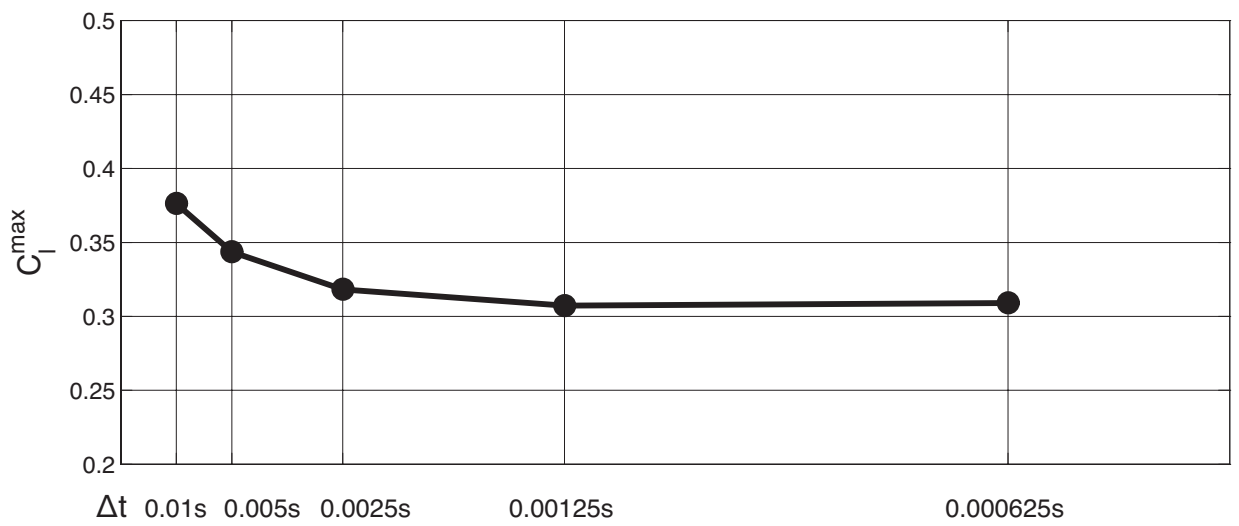

Fig. 7. Convergence of the maximal lift coefficient $C_{1}^{\max }$ for decreasing time step. The horizontal axis is scaled inversely proportional to the time step: $0.01 / \Delta t$. Note that this figure is equivalent to figure $5 \mathrm{~b}$, since $C_{1}^{\max }=-C_{1}^{\min }$ and $C_{1}^{\text {avg }}=0$. 


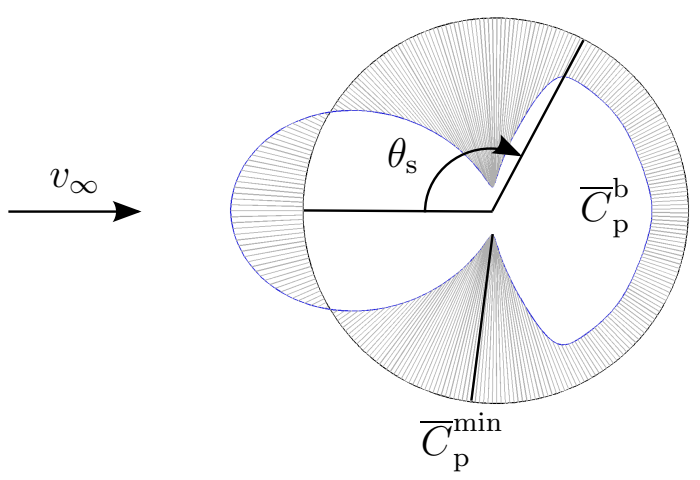

Fig. 8. Time averaged pressure coefficient $\bar{C}_{\mathrm{p}}$ on the circumference of the cylinder with indication of the free stream velocity $v_{\infty}$, the separation angle $\theta_{\mathrm{s}}$, the time averaged base pressure coefficient $\bar{C}_{\mathrm{p}}^{\mathrm{b}}$ and the minimum time averaged pressure coefficient $\bar{C}_{\mathrm{p}}^{\text {min }}$. 


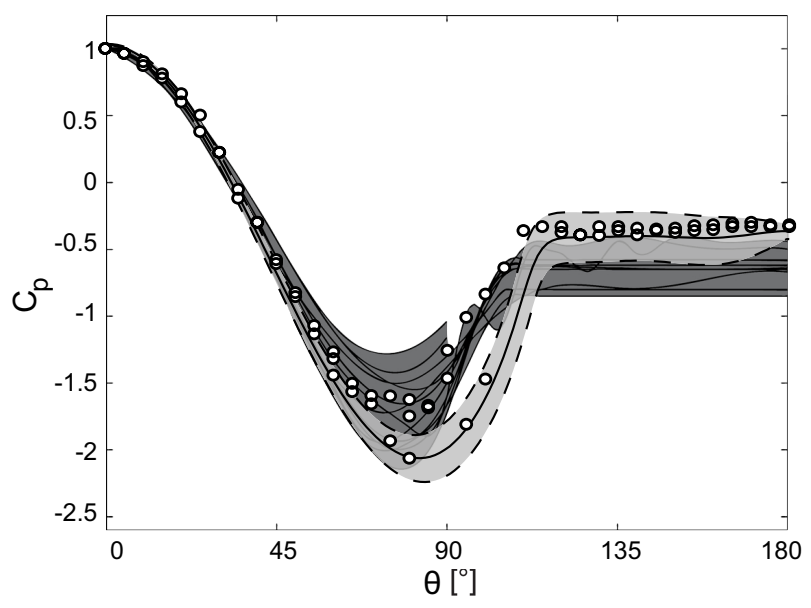

Fig. 9. Comparison of the present calculated $\left(y^{+}=97\right)$ maximal, minimal (dashed lines) and time averaged pressure coefficients $\bar{C}_{\mathrm{p}}(\theta)$ (solid line) for the flow around a single cylinder at $\mathrm{Re}=1.24 \times 10^{7}$ with experimental results of Zdravkovich (1997) for $0.73 \times 10^{7} \leq \operatorname{Re} \leq 3.65 \times 10^{7}$ (dark grey zone) and Shih et al. (1993) at $\operatorname{Re}=0.8 \times 10^{7}$ (०). 
(a)

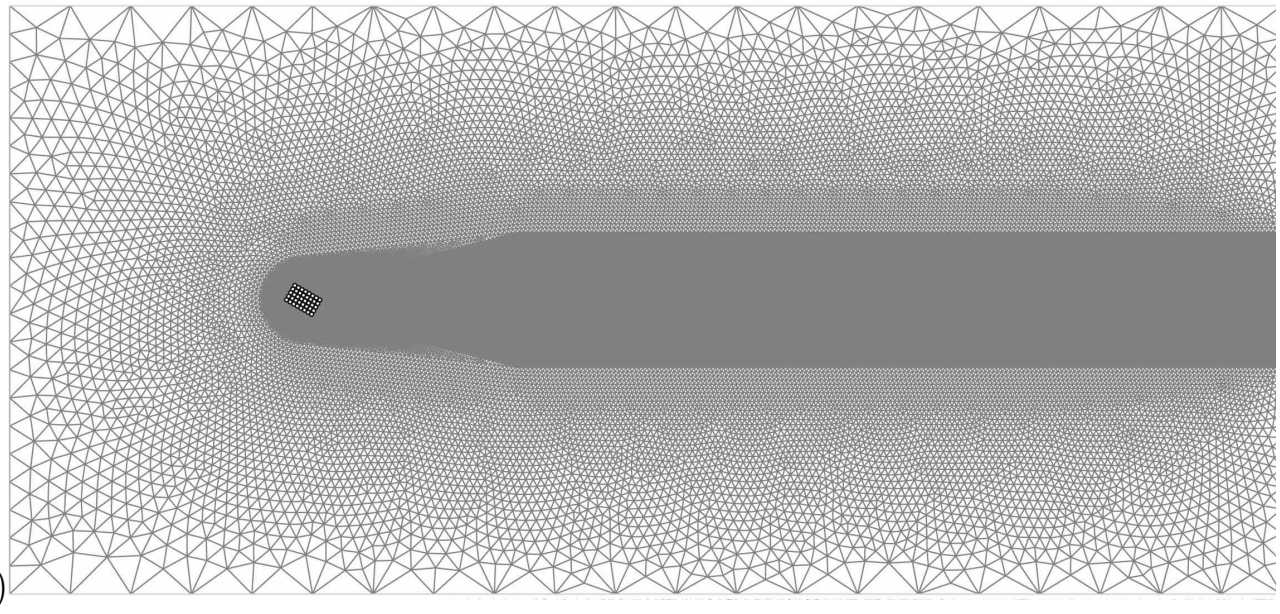

(b)
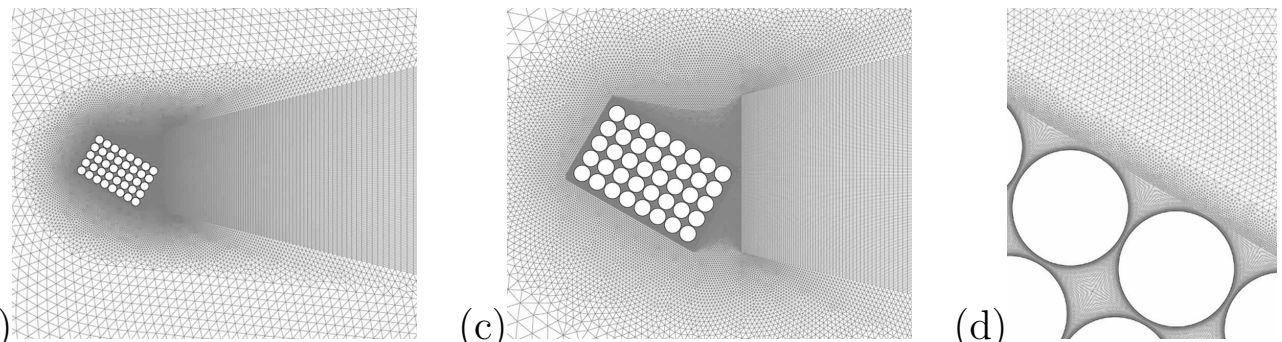

Fig. 10. Computational mesh for the 8 by 5 silo group at an angle of incidence $\alpha=30^{\circ}$, shown for (a) the entire computational domain and details of (b,c) the near-wake region and (d) the near-wall region. 
(a)

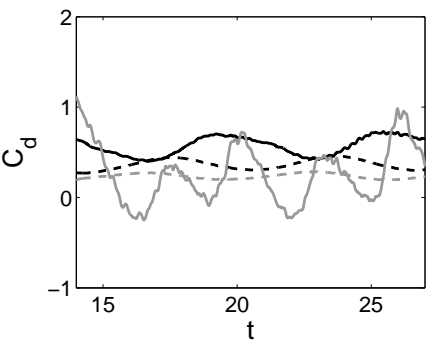

(b)

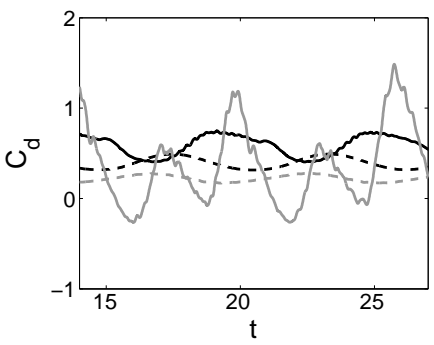

(c)

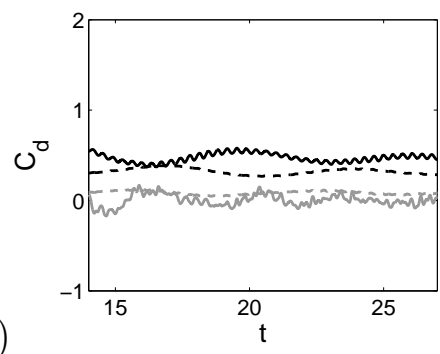

Fig. 11. Time history of drag coefficients for the corner silos of the 8 by 5 silo group (silo 1 - dashed black line; silo 8 - solid black line; silo 33 - dashed grey line; silo 40 solid grey line) with wind flow at angle of incidence $\alpha=30^{\circ}$ and for inlet turbulence intensities of (a) $\mathrm{Tu}=0.1 \%$, (b) $\mathrm{Tu}=1 \%$ and (c) $\mathrm{Tu}=10 \%$. 
(a)

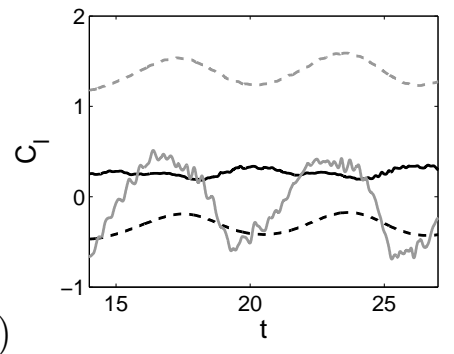

(b)

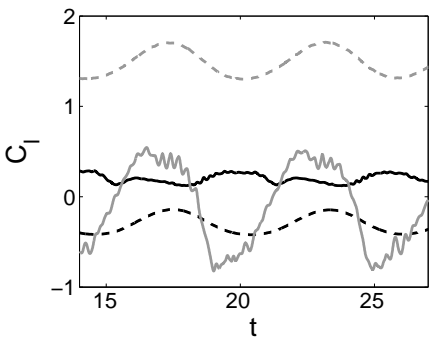

(c)

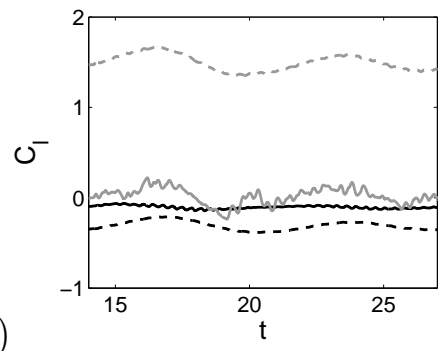

Fig. 12. Time history of lift coefficients for the corner silos of the 8 by 5 silo group (silo 1 - dashed black line; silo 8 - solid black line; silo 33 - dashed grey line; silo 40 solid grey line) with wind flow at angle of incidence $\alpha=30^{\circ}$ and for inlet turbulence intensities of (a) $\mathrm{Tu}=0.1 \%$, (b) $\mathrm{Tu}=1 \%$ and (c) $\mathrm{Tu}=10 \%$. 
(a)

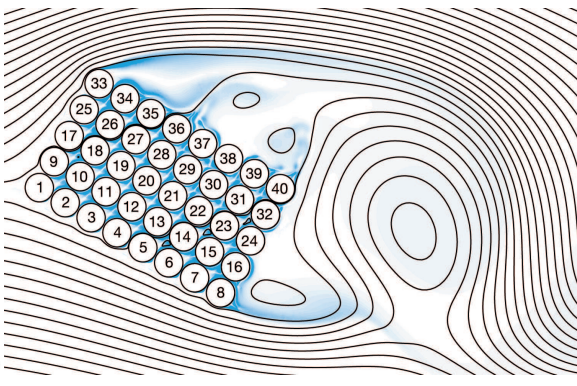

(c)

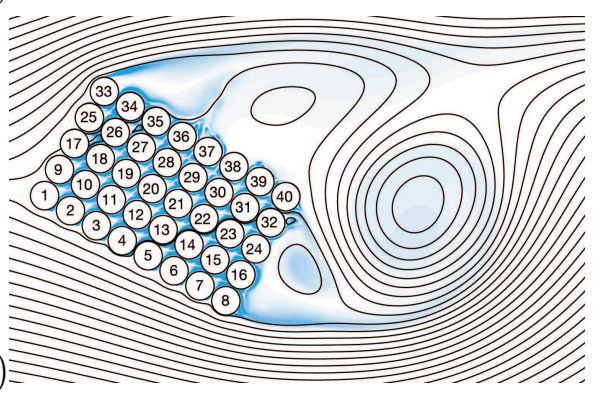

(b)

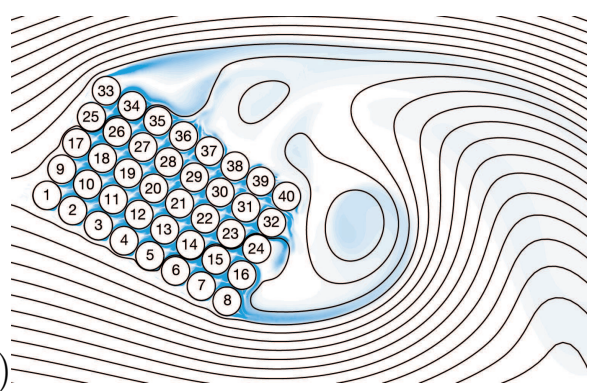

(d)

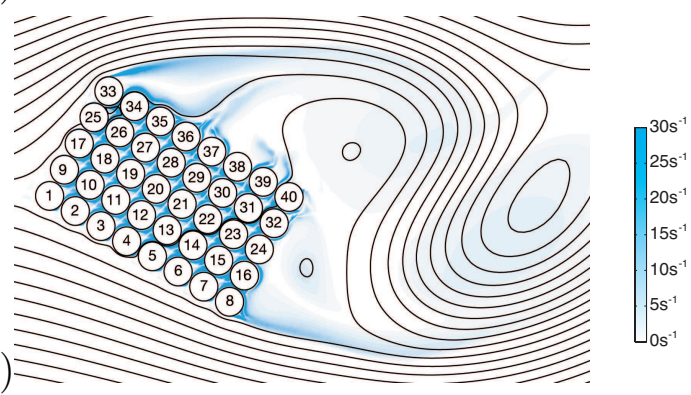

Fig. 13. Velocity streamlines and vorticity contours (filled) of the flow around the 8 by 5 cylinder group for an angle of incidence $\alpha=30^{\circ}$ at (a) $t=77.0 \mathrm{~s}$, (b) $t=78.5 \mathrm{~s}$, (c) $t=80.0 \mathrm{~s}$, and (d) $t=81.5 \mathrm{~s}$. 


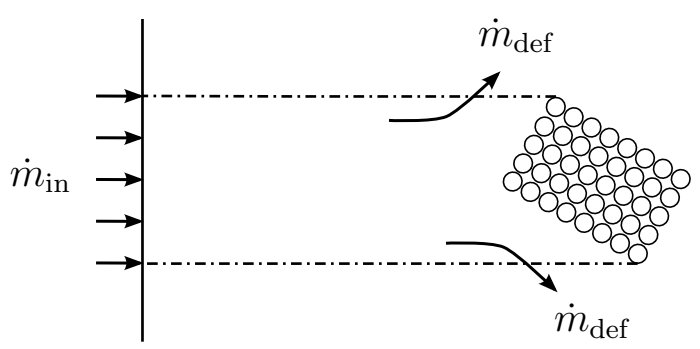

Fig. 14. Schematic representation of the incident mass flow at the inlet of the domain $\dot{m}_{\text {in }}$ and the mass flow deflected around the silo group $\dot{m}_{\text {def }}$. 


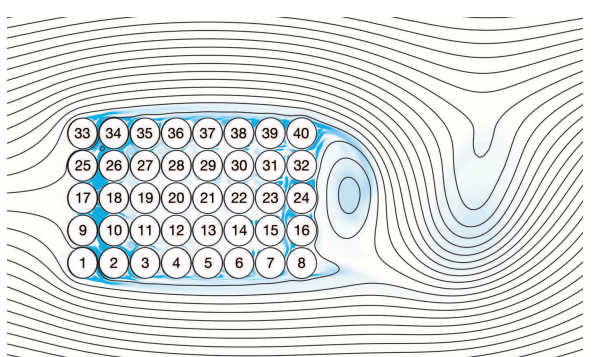

(a)

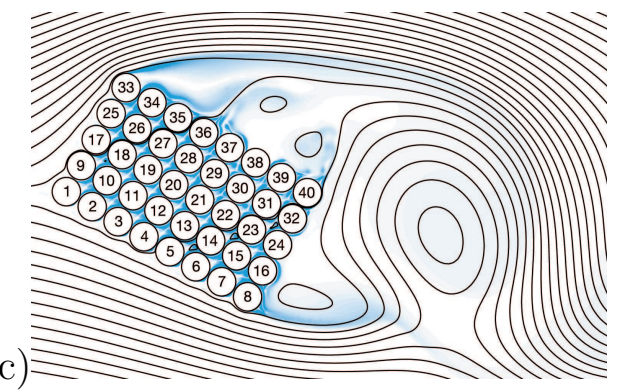

(c)

(e)

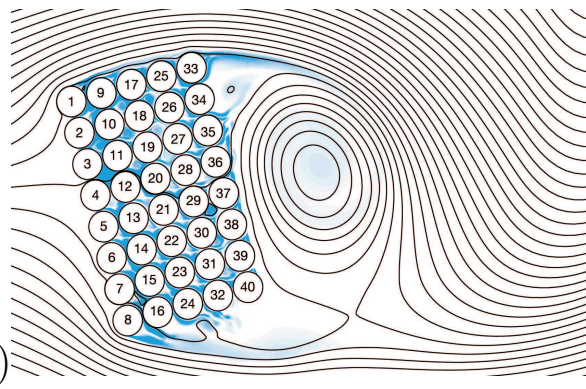

(b)

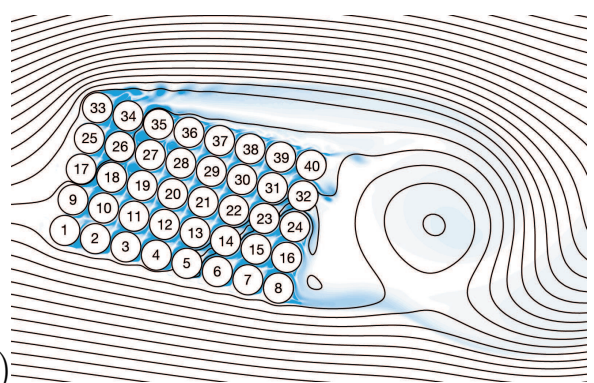

(d)

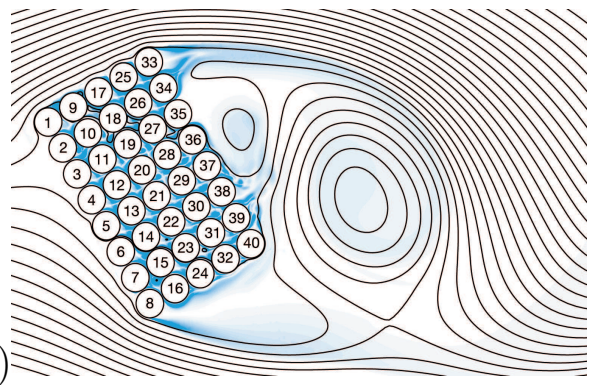

(f)

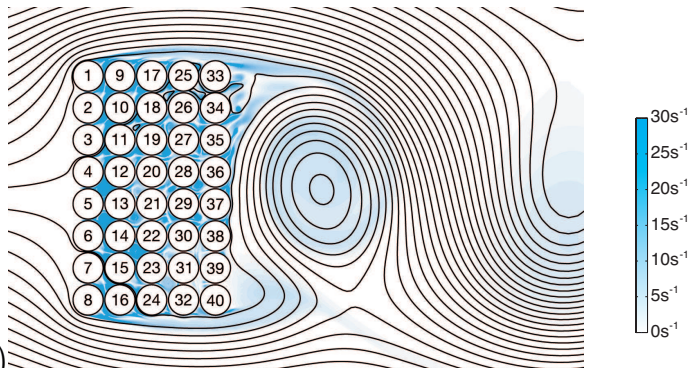

Fig. 15. Velocity streamlines and vorticity contours (filled) of the flow around the 8 by 5 cylinder group for an angle of incidence (a) $\alpha=0^{\circ}$ at $t=80.0 \mathrm{~s}$, (b) $\alpha=15^{\circ}$ at $t=82.5 \mathrm{~s}$, (c) $\alpha=30^{\circ}$ at $t=77.0 \mathrm{~s},(\mathrm{~d}) \alpha=60^{\circ}$ at $t=76.5 \mathrm{~s},(\mathrm{e}) \alpha=75^{\circ}$ at $t=80.5 \mathrm{~s}$, and (f) $\alpha=90^{\circ}$ at $t=83.5 \mathrm{~s}$. 


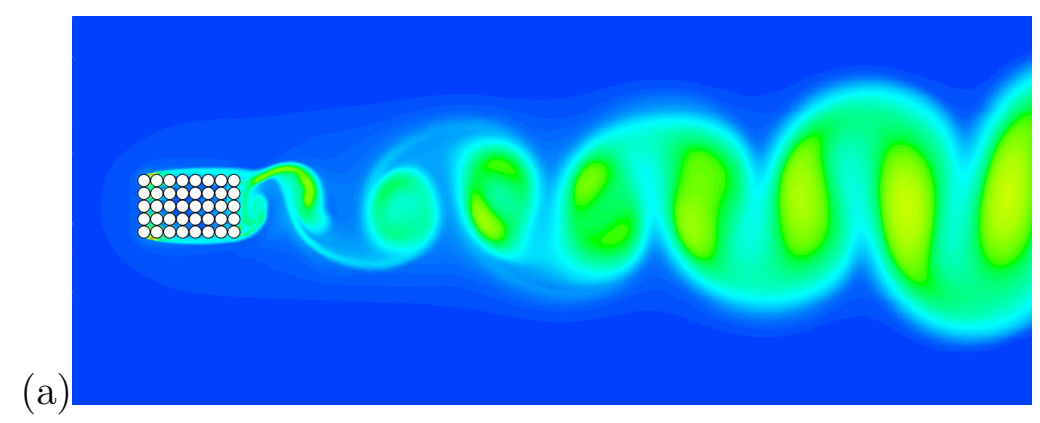

(b)

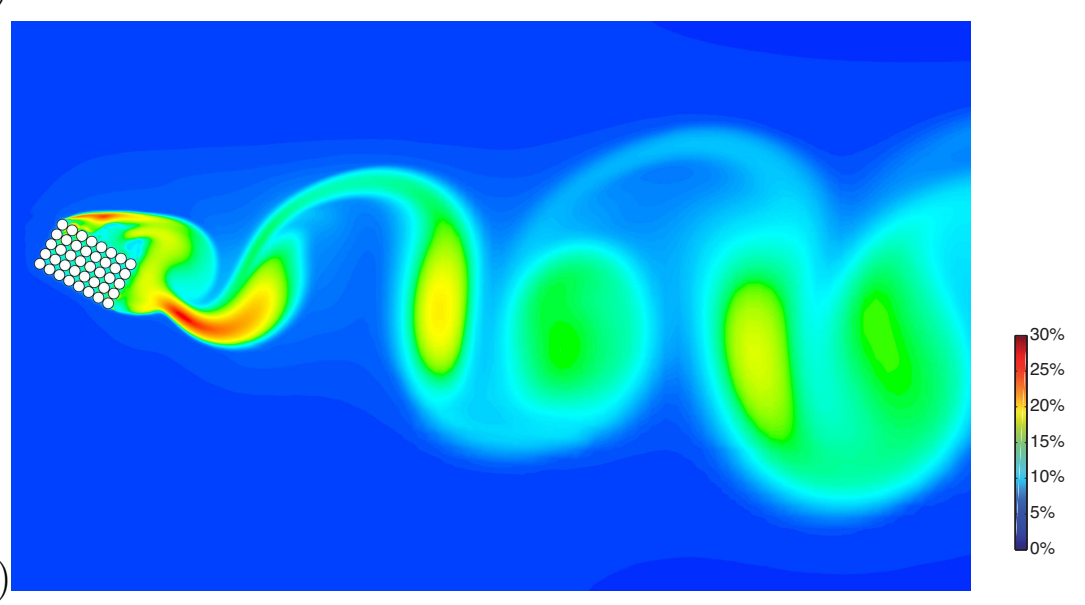

Fig. 16. Turbulence intensity in the flow around the 8 by 5 cylinder group for an angle of incidence (a) $\alpha=0^{\circ}$ at $t=78.5 \mathrm{~s}$ and (b) $\alpha=30^{\circ}$ at $t=77.0 \mathrm{~s}$. 


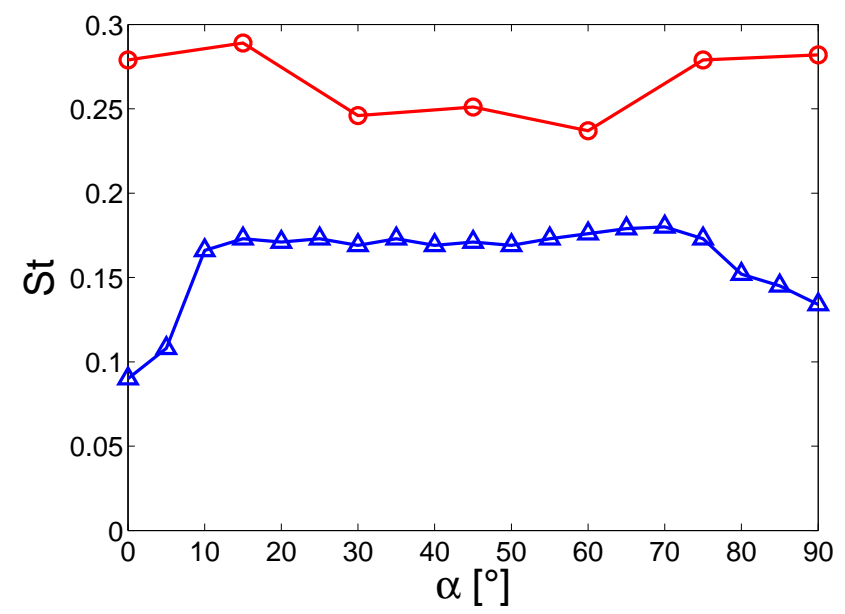

Fig. 17. Comparison of Strouhal numbers for the present 8 by 5 silo group at $\operatorname{Re}=1.24 \times 10^{7}(\circ)$ and a rectangular cylinder with side ratio $L / B=1.67$ at Re between 1.2 and $2.4 \times 10^{4}(\triangle)$ (Knisely, 1990) for angles of incidence $\alpha$ between $0^{\circ}$ and $90^{\circ}$. 

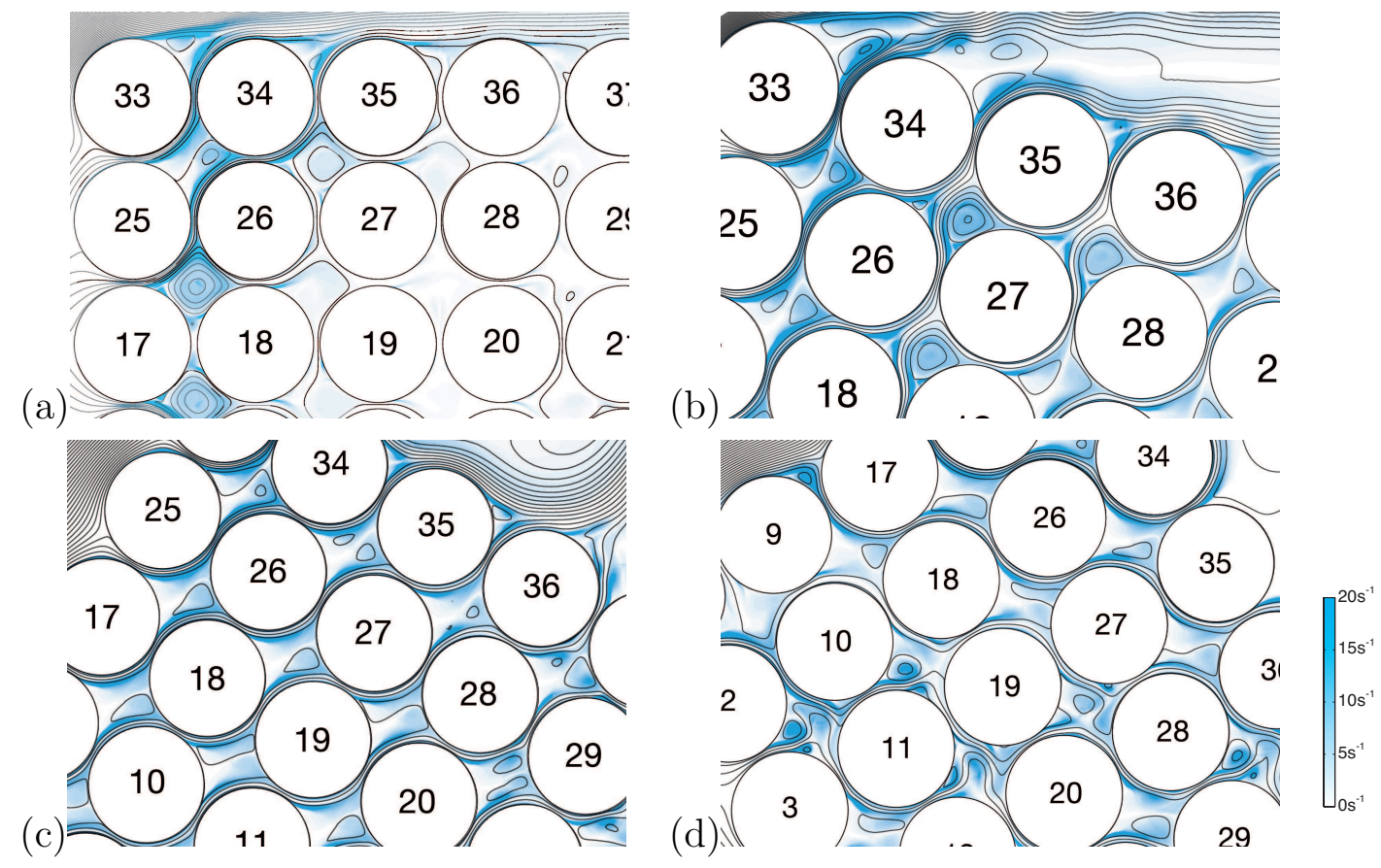

Fig. 18. Detail of velocity streamlines and vorticity contours (filled) for the interstitial space in the 8 by 5 cylinder group for an angle of incidence (a) $\alpha=0^{\circ}$ at $t=78.5 \mathrm{~s}$, (b) $\alpha=15^{\circ}$ at $t=77.0 \mathrm{~s}$, (c) $\alpha=30^{\circ}$ at $t=79.0 \mathrm{~s}$, and (d) $\alpha=60^{\circ}$ at $t=85.0 \mathrm{~s}$. 


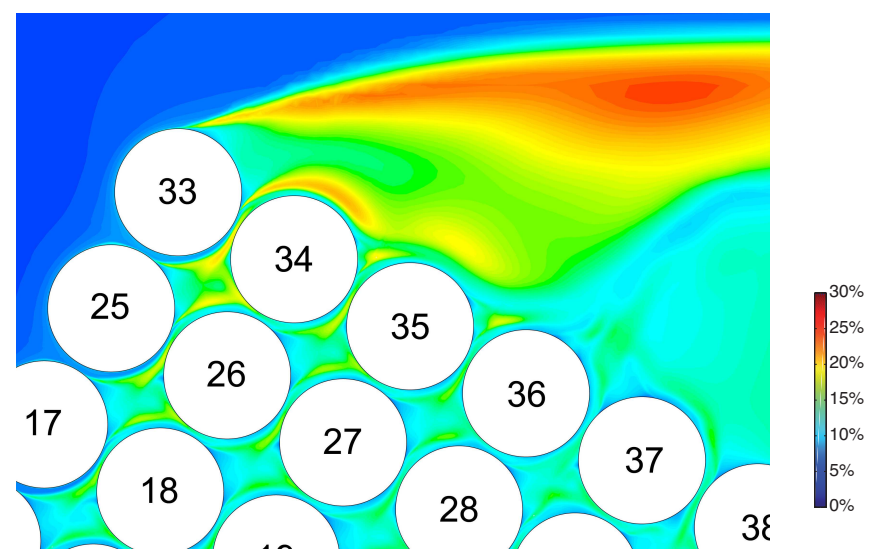

Fig. 19. Contour plot of the turbulence intensity within the cylinder array, for an angle of incidence $\alpha=30^{\circ}$ at $t=77.0 \mathrm{~s}$. 
(a)
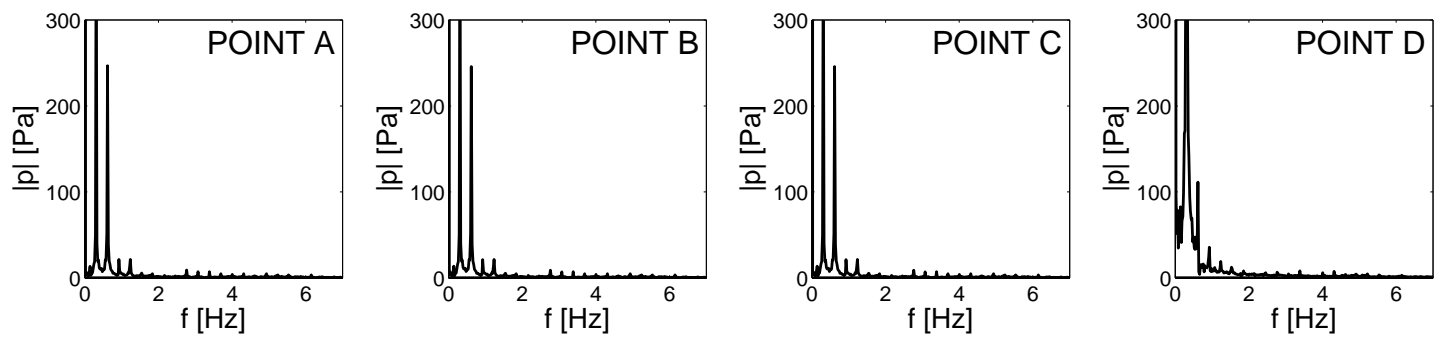

(b)
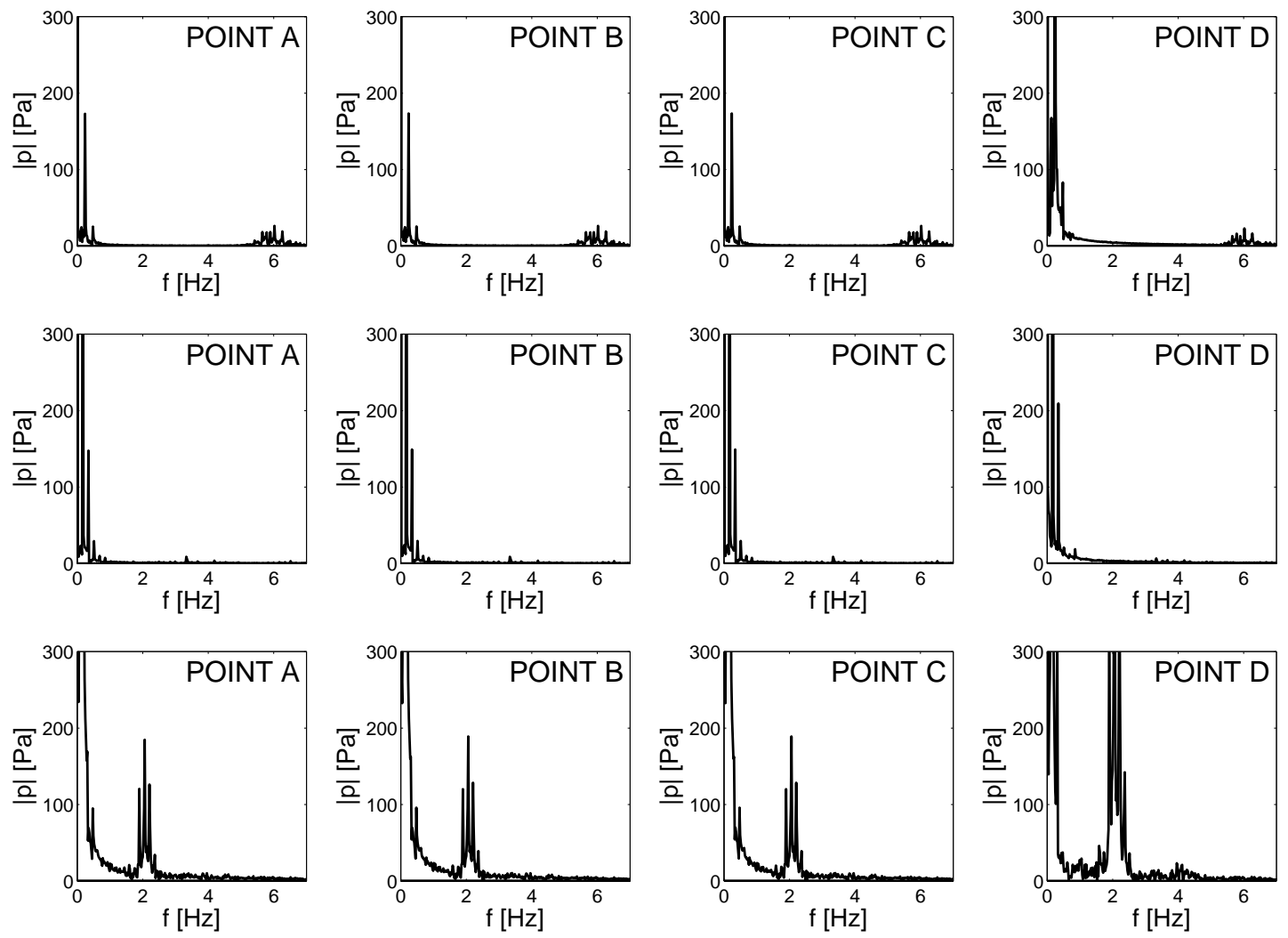

Fig. 20. Frequency spectra for the pressure in points A, B, C and D (figure 2) for angles of incidence (a) $\alpha=0^{\circ}$, (b) $\alpha=15^{\circ}$, (c) $\alpha=30^{\circ}$, and (d) $\alpha=60^{\circ}$. 

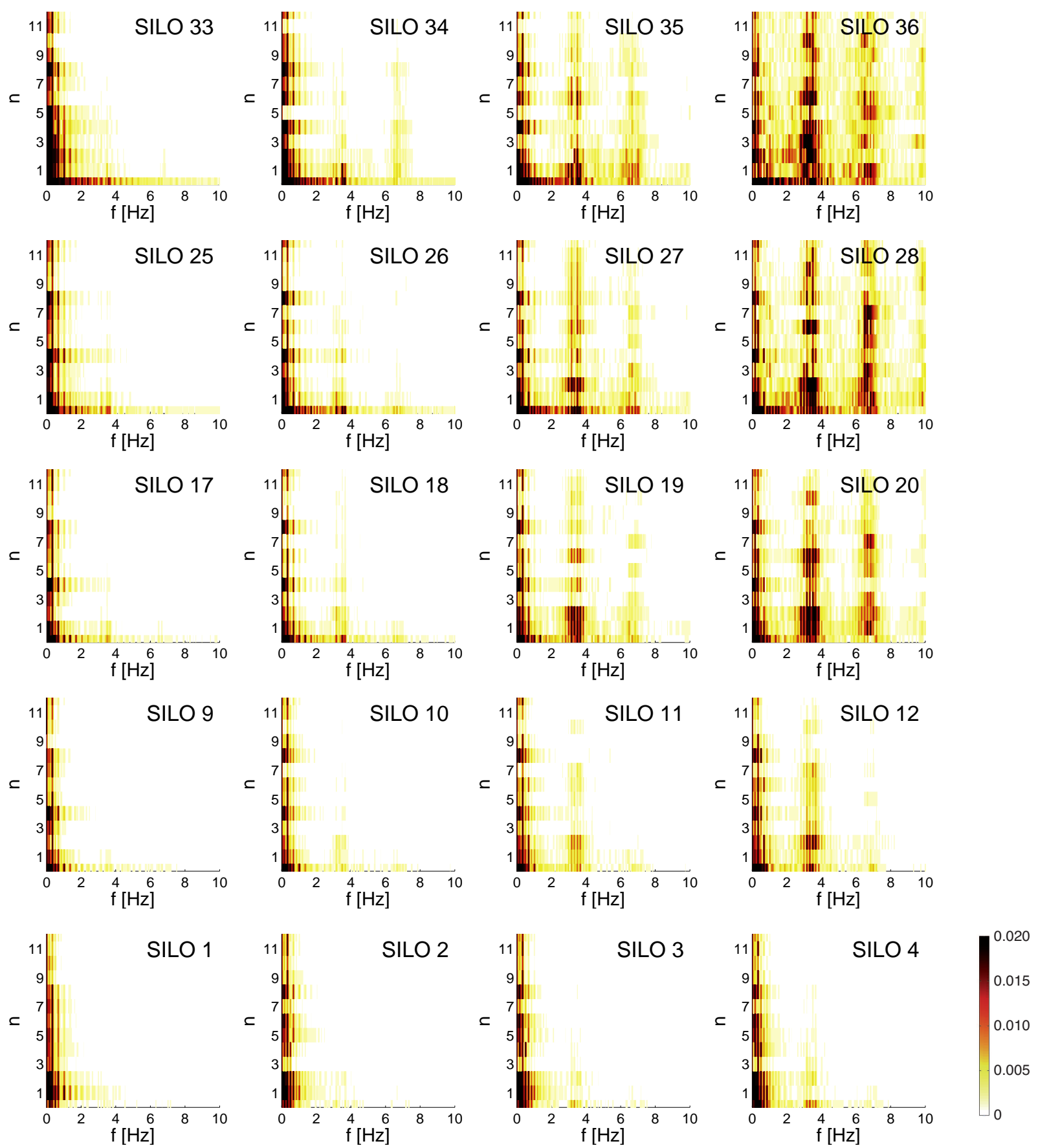

(a)

Fig. 21. Wavenumber-frequency spectra of the amplitude $C_{\mathrm{p}}^{\prime n}(f)$ for angle of incidence $\alpha=30^{\circ}$ : upstream part of the group. 

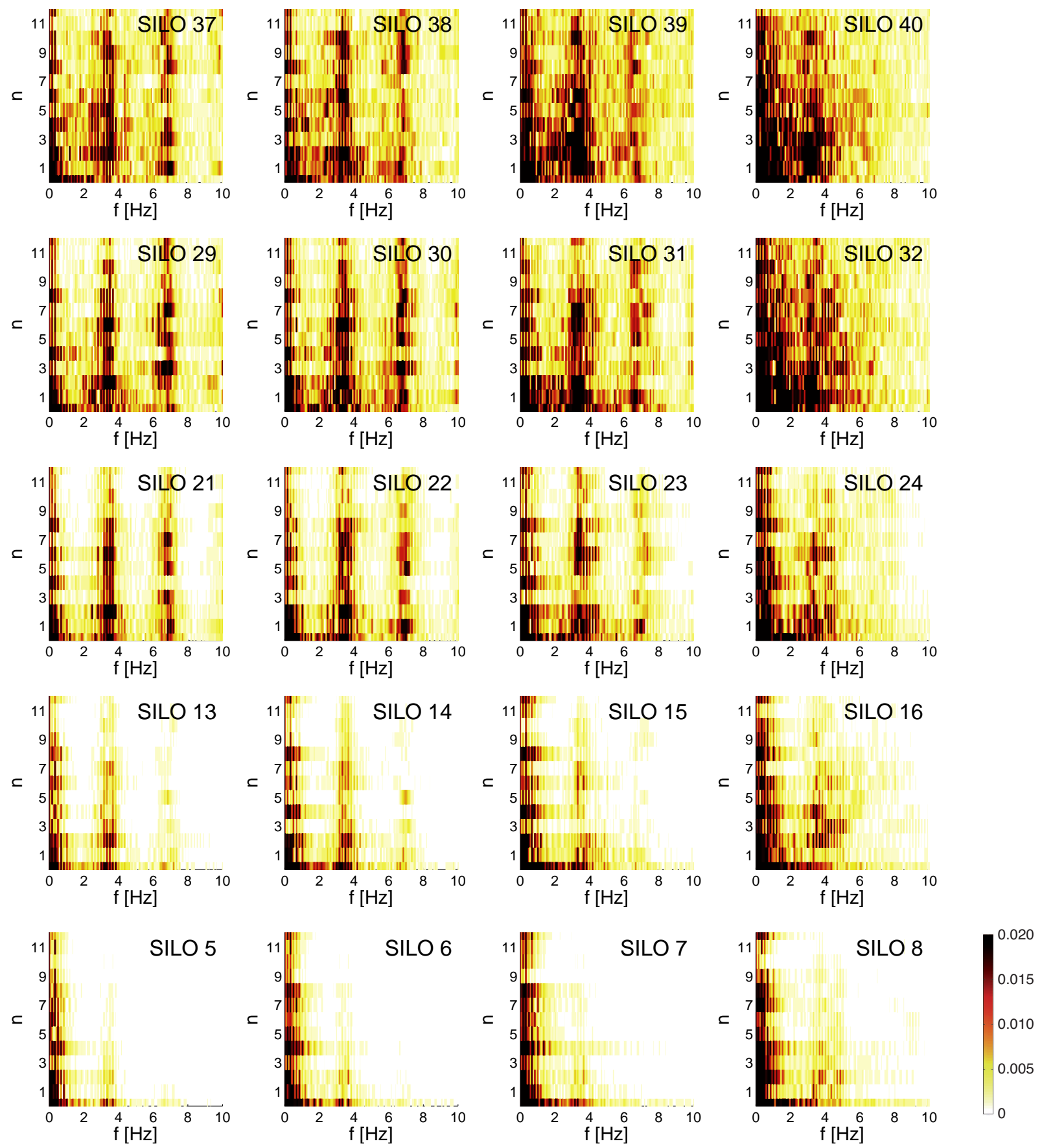

(b)

Fig. 21. Wavenumber-frequency spectra of the amplitude $C_{\mathrm{p}}^{\prime n}(f)$ for angle of incidence $\alpha=30^{\circ}$ : downstream part of the group. 
(a)
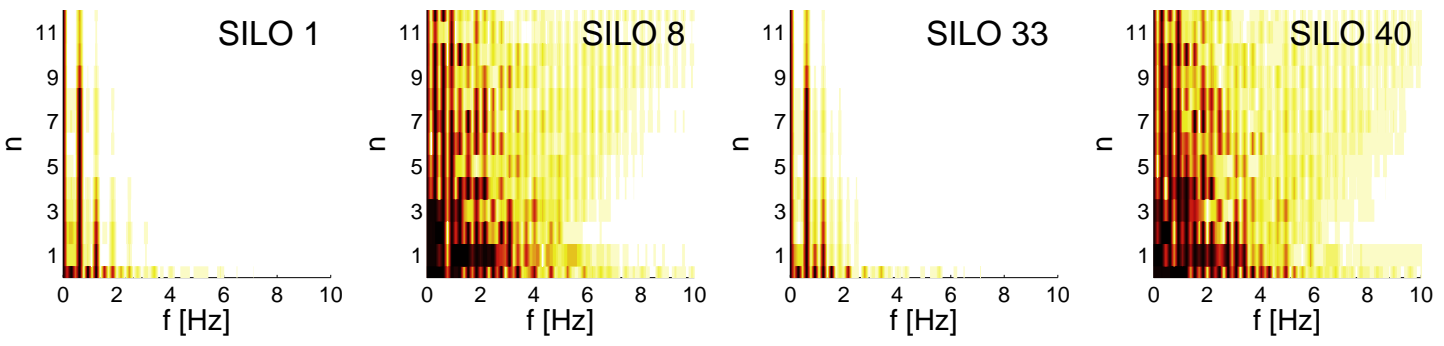

(b)
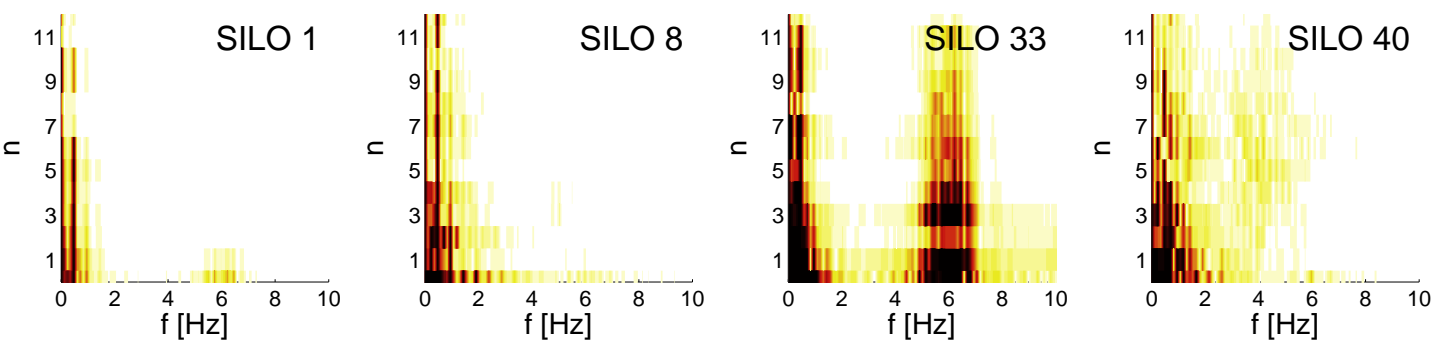

(c)
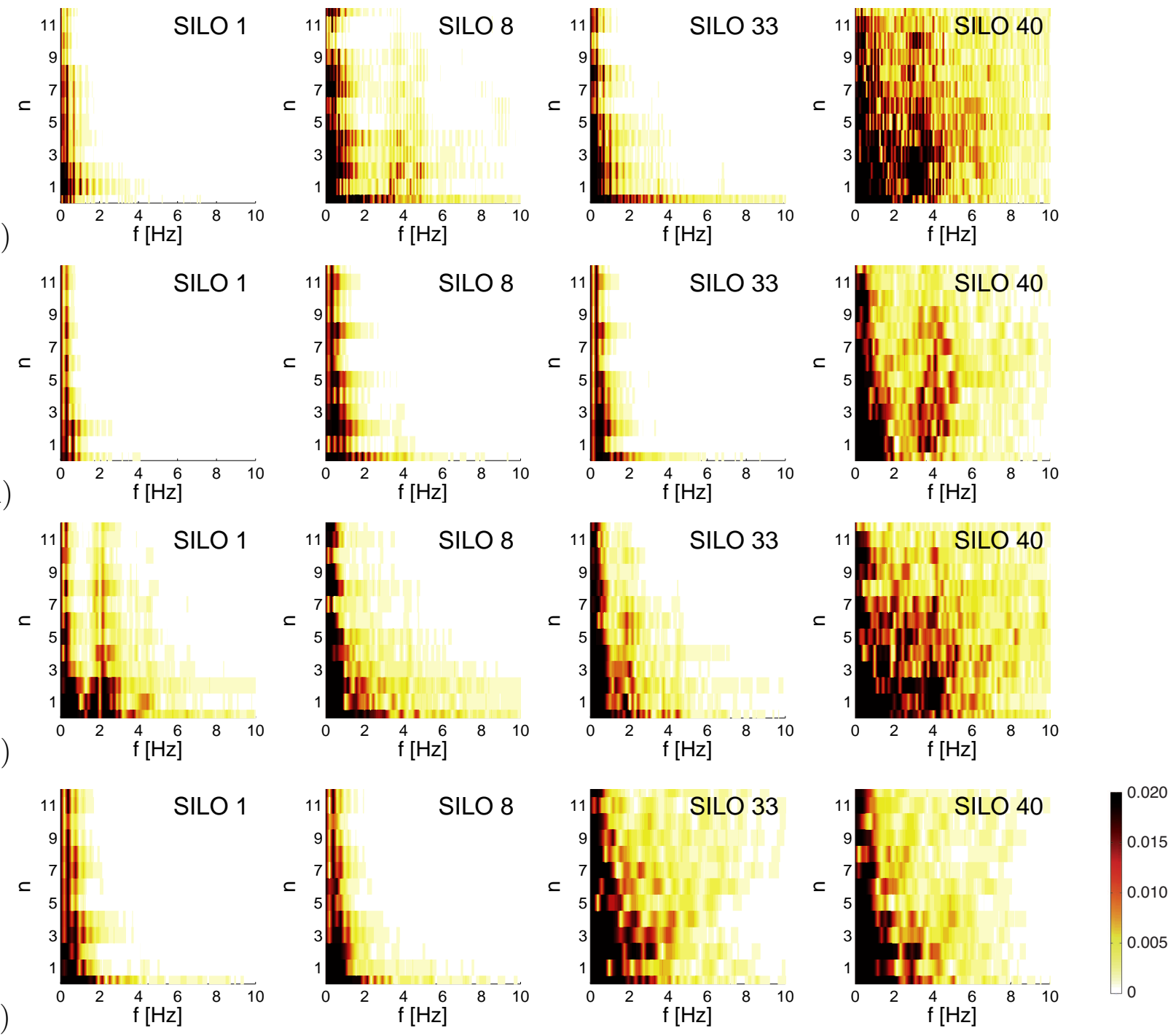

Fig. 22. Wavenumber-frequency spectra of the amplitude $C_{\mathrm{p}}^{\prime n}(f)$ on the corner cylinders $\left(1,8,33\right.$ and 40) of the group for angle of incidence (a) $\alpha=0^{\circ}$, (b) $\alpha=15^{\circ}$, (c) $\alpha=30^{\circ}$, (d) $\alpha=45^{\circ}$, (e) $\alpha=60^{\circ}$ and (f) $\alpha=90^{\circ}$. 


\section{List of Tables}

1 Comparison of the Reynolds number Re, the Strouhal number St, the separation angle $\theta_{\mathrm{s}}$, the averaged base pressure coefficient $\bar{C}_{\mathrm{p}}^{\mathrm{b}}$, the minimum averaged pressure coefficient $-\bar{C}_{\mathrm{p}}^{\text {min }}$ and the averaged drag coefficient $\bar{C}_{\mathrm{d}}$ for the present simulations and experimental and numerical results available in literature.

$2 \quad$ Vortex shedding frequencies $\left(f_{\mathrm{vs}}\right)$ and Strouhal numbers (St) for all different angles of incidence $(\alpha)$ with respective projected width of the silo group $\left(D_{\mathrm{g}}\right)$. 


\begin{tabular}{|c|c|c|c|c|c|c|}
\hline & $\begin{array}{l}\mathrm{Re} \\
{\left[\times 10^{7}\right]}\end{array}$ & $\mathrm{St}$ & $\begin{array}{l}\theta_{\mathrm{s}} \\
{\left[^{\circ}\right]}\end{array}$ & $-\bar{C}_{\mathrm{p}}^{\mathrm{b}}$ & $-\bar{C}_{\mathrm{p}}^{\min }$ & $\bar{C}_{\mathrm{d}}$ \\
\hline \multicolumn{7}{|l|}{ Present numerical simulations } \\
\hline 2D URANS SST, $y^{+}=97$ & 1.24 & 0.32 & 116 & 0.52 & 2.54 & 0.42 \\
\hline 2D URANS SST, $y^{+}=4$ & 1.24 & 0.34 & 116 & 0.49 & 2.62 & 0.39 \\
\hline \multicolumn{7}{|l|}{ Experimental data from literature } \\
\hline Zan (2008) & $0.20-0.50$ & 0.20 & & & & \\
\hline Schewe (1983) & $0.50-0.60$ & 0.27 & & & & 0.52 \\
\hline James et al. (1980) & $0.01-1.09$ & 0.22 & & & & \\
\hline Zdravkovich (1997) & $0.73-3.65$ & $0.27-0.32$ & $100-110$ & $0.5-0.8$ & & $0.4-0.8$ \\
\hline Eurocode 1 - Part 1.4 (BIN, 2005) & 1.00 & 0.18 & 105 & 0.80 & 1.5 & \\
\hline \multicolumn{7}{|l|}{ Numerical simulations from literature } \\
\hline 2D URANS $k-\varepsilon$ transition (Celik and Shaffer, 1995) & 0.36 & & 118 & 0.35 & 2.3 & \\
\hline 2D URANS realizable $k-\varepsilon$ (Holloway et al., 2004) & 1.00 & & 120 & & & 0.26 \\
\hline 2D URANS transition (Holloway et al., 2004) & 1.00 & & 119 & & & 0.25 \\
\hline 2D URANS $k-\varepsilon$ (Saghafian et al., 2003) & 0.84 & 0.25 & 104 & 0.72 & 1.8 & 0.66 \\
\hline 2D URANS nonlinear (Saghafian et al., 2003) & 0.84 & 0.33 & 125 & 1.15 & 2.6 & 0.61 \\
\hline 2D URANS RNG $k-\varepsilon$ (Younis and Przulj, 2006) & 0.35 & 0.28 & 122 & 0.80 & 2.5 & 0.56 \\
\hline 2D URANS modified $k-\varepsilon$ (Younis and Przulj, 2006) & 0.35 & 0.28 & 120 & 1.25 & 2.5 & 0.72 \\
\hline 3D DES (Travin et al., 2000) & 0.30 & 0.35 & 111 & 0.53 & 2.2 & 0.41 \\
\hline 3D DES + curvature (Travin et al., 2000) & 0.30 & 0.33 & 106 & 0.64 & 2.1 & 0.51 \\
\hline 3D URANS $k-\varepsilon$ (Catalano et al., 2003) & 0.10 & 0.31 & & 0.41 & 2.3 & 0.40 \\
\hline 3D LES Smagorinsky (Catalano et al., 2003) & 0.10 & 0.35 & & 0.32 & 2.4 & 0.31 \\
\hline
\end{tabular}

Table 1

Comparison of the Reynolds number Re, the Strouhal number St, the separation angle $\theta_{\mathrm{s}}$, the averaged base pressure coefficient $\bar{C}_{\mathrm{p}}^{\mathrm{b}}$, the minimum averaged pressure coefficient $-\bar{C}_{\mathrm{p}}^{\text {min }}$ and the averaged drag coefficient $\bar{C}_{\mathrm{d}}$ for the present simulations and experimental and numerical results available in literature. 


\begin{tabular}{lllllllll}
\hline$\alpha$ & {$\left[{ }^{\circ}\right]$} & 0 & 15 & 30 & 45 & 60 & 75 & 90 \\
\hline$D_{\mathrm{g}}$ & {$[\mathrm{m}]$} & 28.7 & 38.4 & 45.9 & 50.6 & 52.3 & 50.7 & 46.1 \\
$T_{\mathrm{vs}}$ & {$[\mathrm{s}]$} & 3.23 & 4.18 & 5.85 & 6.32 & 6.93 & 5.70 & 5.14 \\
$f_{\mathrm{vs}}$ & {$[\mathrm{Hz}]$} & 0.31 & 0.24 & 0.17 & 0.16 & 0.14 & 0.18 & 0.20 \\
$\mathrm{St}$ & & 0.28 & 0.29 & 0.25 & 0.25 & 0.23 & 0.29 & 0.29 \\
\hline
\end{tabular}

Table 2

Vortex shedding frequencies $\left(f_{\mathrm{vs}}\right)$ and Strouhal numbers (St) for all different angles of incidence $(\alpha)$ with respective projected width of the silo group $\left(D_{\mathrm{g}}\right)$. 